This item was submitted to Loughborough's Research Repository by the author.

Items in Figshare are protected by copyright, with all rights reserved, unless otherwise indicated.

\title{
Analysis of Ginzburg-Landau-type models of surfactant-assisted liquid phase separation
}

\section{PLEASE CITE THE PUBLISHED VERSION}

https://doi.org/10.1103/PhysRevE.91.032404

\section{PUBLISHER}

(C) American Physical Society

\section{VERSION}

AM (Accepted Manuscript)

\section{PUBLISHER STATEMENT}

This work is made available according to the conditions of the Creative Commons Attribution-NonCommercialNoDerivatives 4.0 International (CC BY-NC-ND 4.0) licence. Full details of this licence are available at: https://creativecommons.org/licenses/by-nc-nd/4.0/

\section{LICENCE}

CC BY-NC-ND 4.0

\section{REPOSITORY RECORD}

Toth, Gyula I., and Bjorn Kvamme. 2019. "Analysis of Ginzburg-landau-type Models of Surfactant-assisted Liquid Phase Separation”. figshare. https://hdl.handle.net/2134/27286. 


\title{
Analysis of Ginzburg-Landau type models of surfactant assisted liquid phase separation
}

\author{
Gyula I. Tóth \\ Institute of Physics and Technology, University of Bergen, \\ Allégaten 55, N-5007 Bergen, Norway and \\ Institute for Solid State Physics and Optics, \\ Wigner Research Centre for Physics, \\ P.O. Box 49, H-1525 Budapest, Hungary* \\ Bjørn Kvamme \\ Institute of Physics and Technology, University of Bergen, \\ Allégaten 55, N-5007 Bergen, Norway
}

(Dated: February 16, 2015)

\begin{abstract}
In this paper the diffuse interface models of surfactant assisted liquid-liquid phase separation are addressed. We start from the generalized version of the Ginzburg-Landau free energy functional based model of van der Sman and van der Graaf. First we analyze the model in the constant surfactant approximation and show the presence of a critical point, at which the interfacial tension vanishes. Then we determine the adsorption isotherms and investigate the validity range of previous result. As a key point of the work, we propose a new model of the van der Sman / van der Graaf type designed for avoiding both unwanted unphysical effects and numerical difficulties being present in previous models. In oder to make the model suitable to describe real systems, we determine the interfacial tension analytically more precisely, and analyze it on the entire accessible surfactant load range. Emerging formulas are then validated by calculating the interfacial tension from the numerical solution of the Euler-Lagrange equations. Time dependent simulations are also performed to illustrate the slowdown of the phase separation near the critical point, and to prove that the dynamics of the phase separation is driven by the interfacial tension.
\end{abstract}

\section{INTRODUCTION}

Adding surfactants, i.e. interface active agents to binary systems consisting of two immiscible fluids may effectively reduce the interfacial tension, thus leading to the formation emulsions [1]. Emulsions play important role in everyday life [2], ranging from medical issues [3, 4] and pharmaceutical materials [5], through cosmetics and food processing [6] to crude oil recovery $[7,8]$. The latter has continuously increasing industrial importance: It has been discovered that alternating water and $\mathrm{CO}_{2}$ injection is a significantly more efficient EOR (Enhanced Oil Recovery) technique than injecting exclusively water or $\mathrm{CO}_{2}[9,10]$, predicting the water $/ \mathrm{CO}_{2}$ emulsion to be an effective material for oil recovery. Some of the possible emulsifiers are promising candidates to form water/hydrocarbon emulsion as well, thus increasing further the recovery rate significantly. This concept would also be economically more advantageous than conventional aquifer $\mathrm{CO}_{2}$ sequestration.

The dynamics of emulsion formation is governed by the microscopic properties of the surfactant loaded liquidliquid interface, which can be addressed by atomisctic simulations. Molecular dynamics simulations provide data for the interfacial properties of the two-phase system on the microscopic level. These data can be then used as input for continuum descriptions addressing mesoscale

\footnotetext{
* Gyula.Toth@ift.uib.no
}

phenomena. Diffuse interface theories are one branch of continuum theories working with space and time continuous order parameter fields. Some of them are based on the Ginzburg-Landau (GL) theory of first-order phase transitions. Such descriptions originate from Gompper and Zschocke [11], and Theissen and Gompper [12]. The latter addresses fluid flow assisted spontaneous emulsification of the oil/water system, while the model of Teramoto and Yonezawa [13] has been successfully used to describe droplet growth dynamics in the same system. Despite their success, these relatively simple phenomenological approaches lack realistic Langmuir and Frumkin adsorption isotherms, therefore a new, more realistic formulation was necessary to be written up. The most widespread version of GL-based surfactant models was published by R. G. M. van der Sman and S. van der Graaf [14], based on the regularization of the surface Diracdelta function of the sharp interface model of Diamant and Andelman [15]. The theory captures the essential effects of surfactants, in particular the lowering of the interfacial tension with increasing surfactant load, and provides promising preliminary results for surfactant laden droplet dynamics in sheared flow. A similar approach was published by Teng, Chern and Lai [16]. Liu and Zhang [17] introduced a generalized model by extending the van der Sman / van der Graaf model with additional free energy terms accounting for lateral interaction between adjacent surfactant layers, as well as asymmetry in the bulk fluids. The new model has been successfully applied for describing the influence of a nontrivial 
phenomena, the Marangoni effect generated by the inhomogeneous interfacial tension on droplet dynamics. A comparative study of the aformentioned models was published by Li and Kim [18]. Despite their efforts, the models still suffered from some unphysical properties, such as shrinking interface width with increasing surfactant load. To avoid the problem, Yun, Li and Kim [19] introduced a non-variatinal formalism of the dynamic equations, however, the surfactant-free solution is not present in the new model anymore. Nevertheless, they have also succesfully addressed the Marangoni effect on droplet dynamics. A different fashion of fixing interface related problems has been proposed by S. Engblom and co-workers [20]. Besides the unphysical behavior of the interface width, the authors gave strong evidences of that the PDE problem has no solution at all under physically relevant circumstances. In order to handle this problem, different surfactant couplings were proposed and analyzed, revealing that the decreasing tendency of the interface width can be reversed together with terminating the instability of the PDE system. This important finding opened the possibility of developing physically consistent diffuse interface models: When in a two-phase liquid/surfactant system the interfacial tension tends to zero at a finite surfactant load, the phase separation critically slows down, thus a mechanically stable emulsion can form. This phenomenon has not yet been addressed in previous works as a function of the surfactant load, and necessitates a detailed analysis of the interfacial tension. Finally we mention that other, non-variational, non GL-based descriptions have also been developed, such as the one by K. E. Teigen [21] and co-workers addressing droplet break-up and coalescence.

The paper is structured as follows. In Section II we introduce a generalized van der Sman / van der Graaf type free energy functional with the corresponding equilibrium (Euler-Lagrange) an dynamic equations. In Section III we analyze the equilibrium solutions: first, using the constant surfactant field approximation, we present simple analytical calculations for the interfacial tension, interface width, and for the speed of phase separation in the different variants of the model, and show how the critical point (i.e. a critical surfactant load at which the interfacial tension vanishes) enters the model. Considering the result we propose a new version of the model in which the surfactant load dependence of the interface width cancels, establishing numerical efficiency. Next, we investigate the existence of the pure (surfactant-free) solution and calculate the adsorption isotherms, then carry out more precise analytical calculations to estimate the interfacial tension as a function of the surfactant load, and analyze the behavior of the model at small surfactant loads and also near the critical point. In the first part of Section IV we briefly discuss the numerical methods used in solving the Euler-Lagrange equations and the dynamic equations. This is followed by the numerical validation of the analytical formula for the interfacial tension. We also perform time dependent simulations and verify the loca- tion of the critical point, together with analyzing the surfactant load dependence of the phase seapration speed. In Section $\mathrm{V}$ we summarize the results.

\section{THE MODEL}

\section{A. Free energy functional}

Following van der Sman and van der Graaf the free energy of an inhomogeneous binary fluid + surfactant system is written as [14]:

$$
F=\int d V\{\mathcal{F}[\phi(\mathbf{r}, t), \psi(\mathbf{r}, t)]\},
$$

where $\phi(\mathbf{r}, t)$ is the liquid-liquid order parameter and $\psi(\mathbf{r}, t)$ the volume fraction of the surfactant, respectively. The integrand reads as

$$
\mathcal{F}=\mathcal{F}_{C H}+\mathcal{F}_{\psi}+\mathcal{F}_{1}+\mathcal{F}_{e x}
$$

where

$$
\begin{aligned}
\mathcal{F}_{C H} & =w g(\phi)+\frac{\kappa}{2}(\nabla \phi)^{2} \\
\mathcal{F}_{\psi} & =\frac{w}{\beta}[\psi \log \psi+(1-\psi) \log (1-\psi)]-w \frac{c}{2} \psi^{2} \\
\mathcal{F}_{1} & =-\psi\left[\lambda_{1} w g(\phi)+\lambda_{2} \frac{\kappa}{2}(\nabla \phi)^{2}\right] \\
\mathcal{F}_{e x} & =w\left(\frac{a}{2} \phi^{2}-e \phi\right) \psi .
\end{aligned}
$$

Here $\mathcal{F}_{C H}$ is the Ginzburg-Landau free energy density of an immiscible Cahn-Hilliard fluid, where $g(\phi)$ is a double-well function $g(\phi)=(1 / 4)\left(1-\phi^{2}\right)^{2}$. The logarithmic term in $\mathcal{F}_{\psi}$ is the ideal part of the entropy of mixing, while the term $-w(c / 2) \psi^{2}$ represents the energy associated with the lateral interaction between adjacent surfactant layers[17]. $\mathcal{F}_{1}$ is a general linear coupling between the liquid-liquid interface and the surfactant field, emerging from the regularization of the surface Diracdelta function [20]. Finally, $\mathcal{F}_{e x}$ accounts for the extra energy due to the presence of the surfactant in the bulk phases [17]. Contrary to the work of Engblom et al [20], we do not consider a coupling term $\propto \psi[\phi(1-\phi)]$ in $\mathcal{F}_{1}$, since it is equivalent to $\propto \psi \phi^{2}$ in $\mathcal{F}_{\text {ex }}$. Note that the only asymmetric term of the free energy functional is $-w e \phi \psi$, being responsible for different equilibrium mole fractions of the surfactant in the bulk phases.

The parameters $w$ and $\kappa$ are related to measurable microscopic quantities, such as the interfacial tension $\left(\sigma_{0}\right)$ and interface width $\left(\delta_{0}\right)$ of the surfactant-free equilibrium liquid-liquid interface via

$$
w=(3 / 2)\left(\sigma_{0} / \delta_{0}\right) \quad \text { and } \quad \kappa=(3 / 4)\left(\sigma_{0} \delta_{0}\right) .
$$

The interface width is defined by the planar interface solution of the Cahn-Hilliard model $\phi^{*}(x)=\tanh \left(x / \delta_{0}\right)$, while the interfacial tension is associated with the parameters via the integral $\sigma_{0}=\int_{-\infty}^{+\infty} d x\left\{\mathcal{F}_{C H}\left[\phi^{*}(x)\right]\right\}$. The 
parameters related to the presence of the surfactant are interpreted as follows: $w / \beta=(R T) / v_{0}$, where $v_{0}$ is the average molar volume of the system, $R$ the gas constant and $T$ the temperature. The model parameter $\beta^{-1}$ then reads as:

$$
\beta^{-1}=\frac{2}{3} \frac{R T}{v_{0}} \frac{\delta_{0}}{\sigma_{0}} .
$$

Furthermore, the model parameter $a$ is responsible for the exclusion of the surfactant in the bulk phases, while $\lambda_{1}$ and $\lambda_{2}$ control the coupling of the surfactant at the liquid-liquid interface. Introducing the lengthscale

$$
\lambda=\sqrt{\kappa /(2 w)}=\delta_{0} / 2
$$

and the free energy scale $H=w \lambda^{D}$, where $D$ is the spatial dimensionality of the problem, results in

$$
\tilde{F}=\int d \tilde{V}\left\{\tilde{\mathcal{F}}_{C H}+\tilde{\mathcal{F}}_{\psi}+\tilde{\mathcal{F}}_{1}+\tilde{\mathcal{F}}_{e x}\right\}
$$

with

$$
\begin{aligned}
\tilde{\mathcal{F}}_{C H} & =g(\phi)+(\tilde{\nabla} \phi)^{2} \\
\tilde{\mathcal{F}}_{\psi} & =\beta^{-1}[\psi \log \psi+(1-\psi) \log (1-\psi)]-\frac{c}{2} \psi^{2} \\
\tilde{\mathcal{F}}_{1} & =-\psi\left[\lambda_{1} g(\phi)+\lambda_{2}(\tilde{\nabla} \phi)^{2}\right] \\
\tilde{\mathcal{F}}_{\text {ex }} & =\psi\left(\frac{a}{2} \phi^{2}-e \phi\right) .
\end{aligned}
$$

Note that the dimensionless surfactant-free interfacial tension and interface width became

$$
\tilde{\sigma}_{0}=4 / 3 \text { and } \tilde{\delta}_{0}=2,
$$

respectively.

\section{B. Euler-Lagrange equations}

The equilibrium solutions represent extrema of the free energy functional with respect to the variables, and can be obtained from the Euler-Lagrange equations:

$$
\frac{\delta \tilde{F}}{\delta \phi}=\tilde{\mu}_{\phi} \quad \text { and } \quad \frac{\delta \tilde{F}}{\delta \psi}=\tilde{\mu}_{\psi},
$$

where $\delta \tilde{F} / \delta \phi$ and $\delta \tilde{F} / \delta \psi$ denote the functional derivatives of $\tilde{F}$ with respect to $\phi$ and $\psi$, respectively:

$$
\begin{aligned}
\frac{\delta \tilde{F}}{\delta \phi}= & \left(1-\lambda_{1} \psi\right)\left(\phi^{3}-\phi\right)+(a \phi-e) \psi \\
& -2 \tilde{\nabla}\left[\left(1-\lambda_{2} \psi\right) \tilde{\nabla} \phi\right] \\
\frac{\delta \tilde{F}}{\delta \psi}= & \frac{1}{\beta} \log \left(\frac{\psi}{1-\psi}\right)-c \psi+\hat{f}[\phi] .
\end{aligned}
$$

Here the operator $\hat{f}[\phi]$ reads as

$$
\hat{f}[\phi]=-\left[\lambda_{1} g(\phi)+\lambda_{2}(\tilde{\nabla} \phi)^{2}\right]+\left(\frac{a}{2} \phi^{2}-e \phi\right) .
$$

Furthermore, $\quad \tilde{\mu}_{\phi}=\left.(\delta \tilde{F} / \delta \phi)\right|_{\phi^{-}, \psi^{-}}$and $\tilde{\mu}_{\psi}=$ $\left.(\delta \tilde{F} / \delta \psi)\right|_{\phi^{-}, \psi^{-}}$are constant background potentials corresponding to the homogeneous background state $\phi(\mathbf{r}) \equiv$ $\phi^{-}$and $\psi(\mathbf{r}) \equiv \psi^{-}$. The planar equilibrium interface solution $\phi^{*}(\tilde{x}), \psi^{*}(\tilde{x})$ can be obtained by solving the 1D Euler-Lagrange equations with the boundary conditions

$$
\begin{aligned}
& \phi^{*}(\tilde{x} \rightarrow \pm \infty) \rightarrow \phi^{ \pm}, \psi^{*}(\tilde{x} \rightarrow \pm \infty) \rightarrow \psi^{ \pm} \\
& {\left[\partial \phi^{*}(\tilde{x}) / \partial \tilde{x}\right]_{\tilde{x} \rightarrow \pm \infty}=\left[\partial \psi^{*}(\tilde{x}) / \partial \tilde{x}\right]_{\tilde{x} \rightarrow \pm \infty} \rightarrow 0}
\end{aligned}
$$

where $\phi^{ \pm}$and $\psi^{ \pm}$can be determined as a function of the surfactant load $\left(\psi_{0}\right)$ from the equilibrium conditions (see later in Section 3.2).

\section{Dynamic equations}

The time evolution of the system is governed by a simple diffusion dynamics:

$$
\begin{aligned}
\tau_{\phi} \frac{\partial \phi}{\partial t} & =\nabla^{2} \frac{\delta F}{\delta \phi} \\
\tau_{\psi} \frac{\partial \psi}{\partial t} & =\nabla \cdot\left[\tilde{M}(\phi) \psi(1-\psi) \nabla \frac{\delta F}{\delta \psi}\right]
\end{aligned}
$$

where the term $\psi(1-\psi)$ in the second equation is necessary to achieve a regular diffusional equation for $\psi$ in the bulk phases, while $\tilde{M}(\phi) \in[0,1]$ prescribes the relative mobility of $\psi$ in the different phases and across the interface. The relaxation times $\tau_{\phi}$ and $\tau_{\psi}$ can be related to diffusion coefficients as follows: Taking the first equation for $\psi(\mathbf{t}, t) \equiv 0$ yields $\partial_{t} \phi=D_{\phi} \nabla^{2}\left[\left(\phi^{3}-\phi\right)-(\kappa / w) \nabla^{2} \phi\right]$, where $D_{\phi}=w / \tau_{\phi}$ is the diffusion coefficient of the phase separating liquid. Note, that this is just the half of the real diffusion coefficient $D_{0}$, since we have to take the equation for $\phi= \pm 1+\delta \phi$, where $|\delta \phi| \ll 1$, yielding the real diffusion equation $\partial_{t} \delta \phi=D_{0} \nabla^{2} \delta \phi$ with $D_{0}=2 D_{\phi}$. Besides, in a bulk phase $(\phi \equiv \pm 1)$ the second equation of motion results in $\partial_{t} \psi=D_{\psi} \nabla^{2} \psi$ (if $\left.c \psi \ll \beta^{-1} \log [\psi /(1-\psi)]\right)$, where $D_{\psi}=\beta^{-1}\left(w / \tau_{\psi}\right)$ is the diffusion constant of the surfactant. Introducing the timescale

$$
\tau=\lambda^{2} \frac{\tau_{\phi}}{w}=\frac{\delta_{0}^{2}}{2 D_{0}}
$$

results in

$$
\begin{aligned}
\frac{\partial \phi}{\partial \tilde{t}} & =\tilde{\nabla}^{2} \frac{\delta \tilde{F}}{\delta \phi} \\
\tilde{\tau}_{\psi} \frac{\partial \psi}{\partial \tilde{t}} & =\tilde{\nabla} \cdot\left[\tilde{M}(\phi) \psi(1-\psi) \tilde{\nabla} \frac{\delta \tilde{F}}{\delta \psi}\right],
\end{aligned}
$$

where

$$
\tilde{\tau}_{\psi}=\frac{\beta^{-1}}{2} \frac{D_{0}}{D_{\psi}} .
$$

For the sake of simplicity, we won't use ? hereafter. 


\section{ANALYSIS}

First we analyze the equilibrium properties of the model. First we calculate the interfacial tension as a function of the surfactant load in the constant surfactant field approximation, i.e. when the spatial variation of the equilibrium emulsifier profile is neglected. We show how the critical point (i.e. where the interfacial tension vanishes) enters the model, and determine the interface width and the dynamic factor (the speed of phase separation), and show how these quantities behave for different surfactant couplings. Next, we analyze the existence criterium of the surfactant-free solution and the adsorption isotherms as a function of the critical point. Finally, we give precise analytical approximation for the interfacial tension in a variant of the model in which the surfactant load dependence of the interface width is cancelled.

\section{A. Constant surfactant field approximation}

Following the method of Engblom et al [20], first we study the model in the constant surfactant field approximation. The simplest case is when the model is symmetric (i.e. $e=0$ ). Since in this case the equilibrium planar interface is represented by an odd function, $\mu_{\phi}=0$ [20]. Therefore, one can write $\phi^{ \pm}= \pm \phi_{0}$ and $\psi^{ \pm}=\psi_{0}$. $\phi_{0}$ can be determined as a function of $\psi_{0}$ from Eq. (6) by introducing $\phi:=\phi_{0} \hat{\phi}$ so that $\phi_{0}$ represents the bulk equilibrium value of $\phi$. Setting $x \rightarrow \pm \infty$, where $\hat{\phi}= \pm 1$ then yields

$$
\phi_{0}^{2}=\frac{1-\left(\lambda_{1}+a\right) \psi_{0}}{1-\lambda_{1} \psi_{0}} .
$$

Note that Eq. (12) is exact for $e=0$, and suggest a critical point

$$
\psi_{c}=\frac{1}{\lambda_{1}+a},
$$

at which $\phi_{0}$ vanishes (as long as $a \neq 0$ ), i.e. no phase separation occurs. Since $\psi_{c}$ is only the function of $\lambda_{1}$ and $a$, but does not depend on the particular form of $F_{\psi}$, the critical point exists in the models of Theissen and Gompper [12], van der Sman and van der Graaf [14], Liu and Zhang [17], Li and Kim [18], Engblom et al [20], and Yun et al [19]. Using Eq. (12) and taking $\psi(x) \equiv \psi_{0}$, Eq. (6) simply becomes the Euler-Lagrange equation of a Cahn-Hilliard model: $\hat{\phi}^{3}-\hat{\phi}=2 \partial_{\hat{x}}^{2} \hat{\phi}$ with the re-scaled length $x=\xi \hat{x}$, where

$$
\xi^{2}=\frac{1-\lambda_{2} \psi_{0}}{1-\left(\lambda_{1}+a\right) \psi_{0}} .
$$

and the planar interface solution can be approximated as:

$$
\phi^{*}(x)=\phi_{0} \tanh [x /(2 \xi)]
$$

Similarly to the Euler-Lagrange equation, using the constant surfactant approximation in the dynamic equation described by Eq. (9) yields: $\partial_{\hat{t}} \hat{\phi}=\hat{\nabla}^{2}\left[g^{\prime}(\hat{\phi})-2 \hat{\nabla}^{2} \hat{\phi}\right]$ with the re-scaled time $t=\hat{t} / s$, where the dynamic factor (defined as the inverse of the timescale) reads as

$$
s=\frac{1-\left(\lambda_{1}+a\right) \psi_{0}}{\xi^{2}} .
$$

The speed of the phase separation can be considered as $v=\frac{d(L / \xi)}{d t}$, where $L$ is the characteristic wavelength of the pattern. Therefore, using the scales yields $v=s \hat{v}$, i.e. the speed of phase separation is proportional to $s$. In the constant surfactant field approximation the interfacial tension reads as:

$$
\sigma=\int_{-\infty}^{+\infty} d x\left\{\mathcal{F}\left[\phi^{*}(x)\right]-\mathcal{F}_{0}\right\}
$$

where $\mathcal{F}\left[\phi^{*}(x)\right]$ is the integrand of Eq. (4) evaluated for Eq. (15) and $\psi^{*}(x)=\psi_{0}$, while $\mathcal{F}_{0}=$ $\mathcal{F}\left[\phi^{*}(x), \psi^{*}(x)\right]_{x \rightarrow-\infty}$. The relative interfacial tension then reads as

$$
\kappa:=\frac{\sigma}{\sigma_{0}}=\xi \phi_{0}^{2}\left[1-\left(\lambda_{1}+a\right) \psi_{0}\right],
$$

where $\sigma_{0}=4 / 3$ is the interfacial tension of the surfactant-free system. Note that Eqns. (16) and (18) report both the phase separation speed and the interfacial tension to vanish at the critical point. Fig 1 shows the relative interface width $\left[\xi=\left(1-d \psi_{0}\right)^{y_{\xi}}\right]$, the dynamic factor $\left[s=\left(1-d \psi_{0}\right)^{y_{s}}\right]$, and the relative interfacial tension $\left[\kappa=\left(1-d \psi_{0}\right)^{y_{\kappa}}\right]$ as a function of the surfactant load in the constant surfactant field approximation for different surfactant field couplings (see Table I) in case of $\lambda_{1}+\lambda_{2} \gg a$ and for $\psi_{c}<1$. In this case the surfactant load dependence of the interface width is significant: The original model using the regularization of the surface Dirac-delta function $(\nabla \phi)^{2}$ results in unphysical behavior, namely, the interface width vanishes together with the divergence of the speed of phase separation. In contrast, the regularization proposing $g(\phi)$ gives a more physical result, since the speed of phase separation decreases with decreasing interfacial tension together with increasing interface width. Unfortunately, however, the critical point is practically inaccessible numerically, because of the divergent interface width: even an infinitesimal difference in the surfactant load can result in orders of magnitude change of the interface width. To resolve this problem, we propose a variant of the model where $\lambda_{1}=d$ and $\lambda_{2}=a+d$, yielding a constant interface width, independently from the model parameters $a, d$, $c$ and $\beta$. Thus, the entire $\psi_{0}=0 \ldots \psi_{c}$ range becomes accessible in one single simulation, which becomes important when one wants to address the migration of the surfactant from loaded places to empty regimes, for example. 

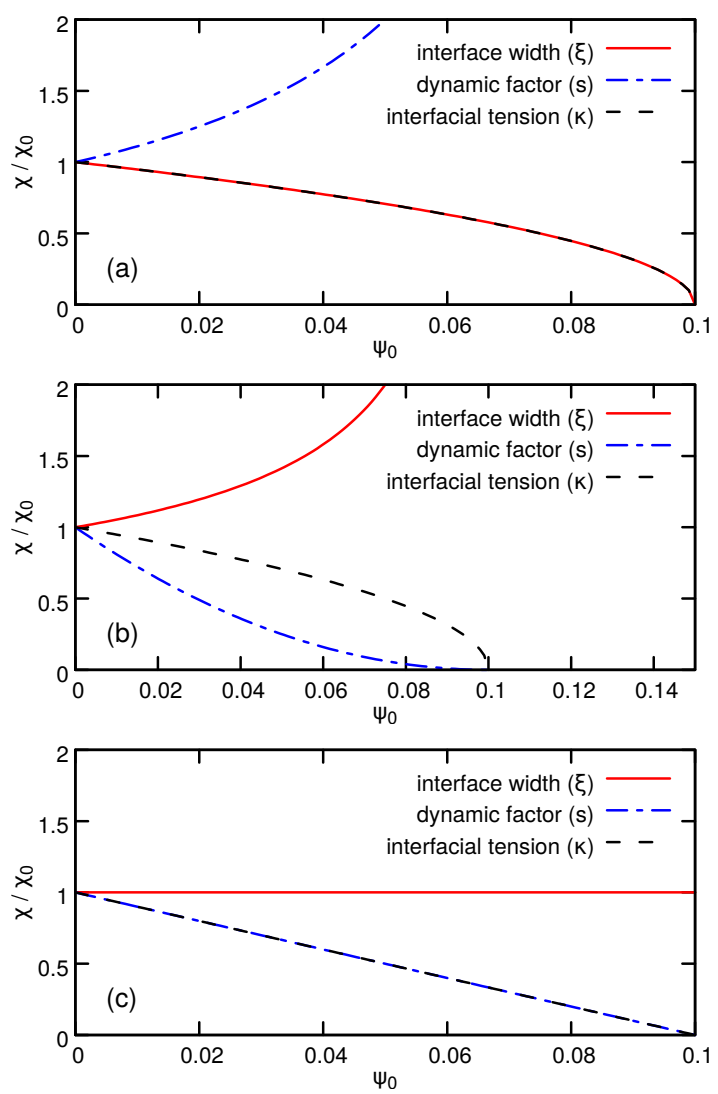

FIG. 1. Relative interface width, phase separation speed (dynamic factor) and interfacial tension as a function of the surfactant load in the constant surfactant field approximation in the case of (a) the pure gradient $\left(\lambda_{1}=0, \lambda_{2}=d\right)$, (b) the fourth order polynomial $\left(\lambda_{1}=d, \lambda_{2}=0\right)$, and (c) the proposed $\left(\lambda_{1}=d, \lambda_{2}=a+d\right)$ surfactant field couplings. (See also Table 1.)

TABLE I. Exponents of the relative interface width $[\xi=$ $\left.\left(1-d \psi_{0}\right)^{y_{\xi}}\right]$, dynamic factor $\left[s=\left(1-d \psi_{0}\right)^{y_{s}}\right]$ and relative interfacial tension $\left[\kappa=\left(1-d \psi_{0}\right)^{y_{\kappa}}\right]$ in case of $\lambda_{1}, \lambda_{2} \gg a$ and $\psi_{c} \leq 1$ for different surfactant couplings.

\begin{tabular}{ccccc}
\hline$\lambda_{1}$ & $\lambda_{2}$ & $y_{\xi}$ & $y_{s}$ & $y_{\kappa}$ \\
\hline 0 & $\mathrm{~d}$ & $1 / 2$ & -1 & $1 / 2$ \\
$\mathrm{~d}$ & 0 & $-1 / 2$ & 2 & $1 / 2$ \\
$\mathrm{~d}$ & $\mathrm{a}+\mathrm{d}$ & 0 & 1 & 1 \\
\hline
\end{tabular}

\section{B. Adsorption isotherms}

\section{Existence of the $\psi \equiv 0$ solution}

Considering Eq. (7) it is obvious that $\delta F / \delta \psi=\mu_{\psi}$ is an algebraic equation, yielding the $1 \mathrm{D}$ equilibrium profile $\psi^{*}(x)$ in the following implicit form:

$$
\psi^{*}(x)=\frac{\psi^{-}}{\psi^{-}+\left(1-\psi^{-}\right) \exp \left\{\beta \Delta \hat{f}\left[\phi^{*}(x)\right]-c \Delta \psi^{*}(x)\right\}},
$$

where $\Delta \psi(x)=\psi^{*}(x)-\psi^{-}$and

$$
\Delta \hat{f}\left[\phi^{*}(x)\right]=\hat{f}\left[\phi^{*}(x)\right]-\hat{f}\left[\psi^{-}\right] .
$$

Note that $\psi^{*}(x) \equiv 0$ is a solution of Eq. (19), since $\psi^{-}=\Delta \psi^{*}(x)=0$, and $\beta \Delta f\left[\phi^{*}(x)\right]$ is bounded for $\phi^{*}(x)=\tanh (x / 2)$. In contrast, this does not apply for models containing no ideal mixing term in $F_{\psi}$ : In the model of Theissen and Gompper $\mu_{\psi}=s \psi-g_{\psi} \nabla^{2} \psi+\hat{f}[\phi]$ with $\hat{f}[\phi]=\gamma_{1} \phi^{2}+\gamma_{2} l^{2}(\nabla \phi)^{2}+\gamma_{3} l^{4}\left(\nabla^{2} \phi\right)^{2}[12]$, yielding the 1D Euler-Lagrange equation $\hat{f}\left[\phi^{*}(x)\right]=\hat{f}\left[\phi_{b}\right]$ for the surfactant-free solution $\phi^{*}(x)=\phi_{b} \tanh (x / \xi)$. Since $\gamma_{1,2,3}$ can be arbitrary, $f\left[\phi^{*}(x)\right]=\hat{f}\left[\phi_{b}\right]$ does not apply in general, therefore, the surfactant-free equilibrium planar interface is not a solution of the problem in principle. The derivation can be repeated in the case of the model of $\mathrm{Li}$ and $\mathrm{Kim}$ [18], yielding $\partial_{x} \phi^{*}(x)=0$ for the surfactant free planar interface $\phi^{*}(x)=\tanh (x / \xi)$, which is definitely not true. These cases shed light on a general problem: The reduction of the free energy functional to the Cahn-Hilliard model is necessary, but not sufficient for the surfactant-free planar interface to be the solution of the general model in case of $\psi \equiv 0$. The reason of this is that reducing the free energy first then solving the EL equation(s) is identical to a conditional extremum problem, but a conditional extremum is not necessarily the extremum of the general problem at all. Finally we mention that this discrepancy resulted in unrealistic adsorption isotherms in the aformentioned models, where the adsorbed amount of surfactant at the interface does not vanish even for zero far-field surfactant load.

\section{Langmuir and Frumkin adsorption isotherms for $c=0$}

Besides ensuring the existence of the pure equilibrium planar interface solution, Eq. (19) plays one other important role: for $e=0$ and $c=0$ the adsorption isotherm reads as:

$$
\psi_{a}\left(\psi_{0}\right)=\frac{\psi_{0}}{\psi_{0}+\left(1-\psi_{0}\right) \exp \left[\beta \theta\left(\psi_{0}\right)\right]},
$$

where $\psi_{a}\left(\psi_{0}\right)=\left.\psi^{*}(x)\right|_{x=0}$ is the surfactant mole fraction at the interface as a function of the bulk surfactant load $\psi_{0}$, and $\theta\left(\psi_{0}\right)=\left.\Delta \hat{f}\left[\phi^{*}(x)\right]\right|_{x=0}$, namely:

$$
\theta\left(\psi_{0}\right)=\left(\frac{\phi_{0}}{2 \xi}\right)^{2}\left\{\xi^{2}\left[\lambda_{1}\left(\phi_{0}^{2}-2\right)-2 a\right]-\lambda_{2}\right\} .
$$

Considering Eqns. (12) and (14) one can identify 3 characteristic points of the $\psi_{a}\left(\psi_{0}\right)$ curve: As long as $\theta\left(\psi_{0}\right)$ is bounded on $\psi_{0} \in[0,1]$,

$$
\psi_{a}(0)=0 \quad \text { and } \quad \psi_{a}(1)=1
$$


apply. Moreover, since $\theta\left(\psi_{c}\right)=0$, a third characteristic point also exists, namely

$$
\psi_{a}\left(\psi_{c}\right)=\psi_{c} .
$$

Since the model has the absolute scale $\psi_{0} \in[0,1]$, there are two essentially different cases:

- Previous works typically considered $\psi_{c}>1[14,20]$, for which we have only the first two characteristic points together with $\psi_{a}\left(\psi_{0}\right) \in[0,1]$ on $\psi_{0} \in[0,1]$. In this case, the Langmuir isotherms can be derived from Eq. (21) as follows: If $\theta\left(\psi_{0}\right) \approx \theta(0)$, and $\exp [\beta \theta(0)]$ is small enough to approximate the prefactor as $1-\psi_{0} \approx 1$, the well-known Langmuir adsorption isotherm

$$
\psi_{a} \approx \frac{\psi_{0}}{\psi_{0}+\Psi}
$$

emerge with $\Psi=\exp [\beta \theta(0)]$. Converting the model parameters used by both van der Sman and van der Graaf [14] and Engblom et al for "Model 0" [20] results in $a=1 / 2, \lambda_{1}=0, \lambda_{2}=1$, and $\beta \gtrsim 8$, respectively. Therefore, $\psi_{c}=2$ was used in their work, while $\theta(0)=-1 / 2$ and $\exp [\beta \theta(0)] \lesssim 0.018$ was small enough to use Eq. (23) on almost the entire range $\psi_{0} \in[0,1]$ (see Fig 2.a). We note, that Eq. $\quad(22)$ starts as $\theta\left(\psi_{0}\right)=-(1 / 2)+(1 / 8) \psi_{0}+$ $O\left(\psi_{0}^{2}\right)$, therefore we have

$$
\psi_{a}\left(\psi_{0}\right)=\frac{\psi_{0}}{\psi_{0}+\Psi \exp \left(\beta \theta_{1} \psi_{0}\right)}
$$

in the first order, which becomes a Frumkin adsorption isotherm for lateral interaction parameters strong enough to win over $\theta_{1} \psi_{0}$, namely, $c\left(\psi_{a}-\psi_{0}\right)>\theta_{1} \psi_{0}$ [see Eq. (19)], as also suggested by Engblom et al [20].

- In the present work we focus on $\psi_{c}<1$. Fig 2.b shows the breakdown of the Langmuir adsorption isotherm for $\psi_{0} \approx \psi_{c}$, indicating that Eq. (21) must be considered instead of Eq. (23). For the sake of interest, we mention that, although $\psi_{a}\left(\psi_{0}\right) \in$ $[0,1]$ for $\psi_{0} \in[0,1]$ still applies, $\psi_{a}\left(\psi_{0}\right) \in\left[0, \psi_{c}\right]$ does not necessarily apply for $\psi_{0} \in\left[0, \psi_{c}\right]$. This means that it is possible to overload the interface for $\psi_{0} \in\left(0, \psi_{c}\right)$ in principle, however, $\psi_{a}\left(\psi_{c}\right)=$ $\psi_{c}$ is still valid. This can be seen on Fig. 5. of Engblom et al [20]: For "Model 2" the parameters read as $a=1$ and $\lambda_{1}=\lambda_{2}=0$, yielding $\psi_{c}=$ 1. For Model "3", $a=1 / 2, \lambda_{1}=1$ and $\lambda_{2}=0$, indicating $\psi_{c}=2 / 3$. The Langmuir isotherms give reasonable estimation for the absorbed amount of the surfactant at the interface for $\psi_{0}<0.1$, which is far from the critical value in both cases, and it is obvious that the interface load can be higher than $2 / 3$ in case of "Model 3".

Finally, we give the general condition of adsorption. The Taylor expansion of Eq. (21) [and also that of Eq.
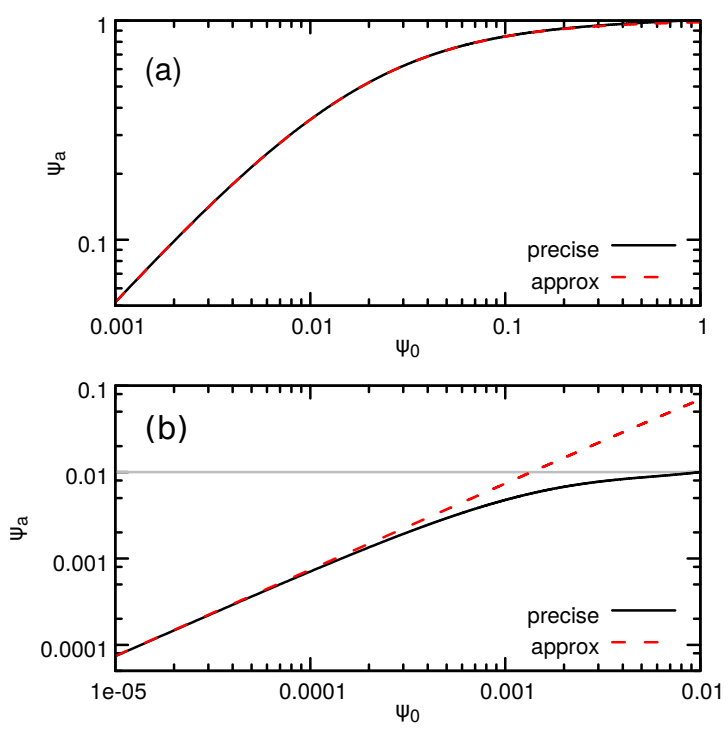

FIG. 2. Adsorption isotherms from Eq. (21) (solid black) and Eq. (23) (dashed red) in case of $\psi_{c}=2$ (panel a) and $\psi_{c}=0.01$ (panel b). The corresponding model parameters read as $a=1 / 2, \lambda_{1}=0, \lambda_{2}=1$, and $\beta=8$ for panel a [14], and $a=201, \lambda_{1}=-101, \lambda_{2}=100$, and $\beta=1 / 120$ for panel b, respectively. Note the strong error of the Langmuir isotherm described by Eq. (23) on panel b. for $\psi_{0} / \psi_{c} \gtrsim 0.1$.

(23)] yields $\psi_{a}\left(\psi_{0}\right)=\exp [-\beta \theta(0)] \psi_{0}+O\left(\psi_{0}^{2}\right)>\psi_{0}$, from which $\theta(0)<0$ follows, indicating

$$
2 a+\lambda_{1}+\lambda_{2}>0,
$$

as the general condition for adsorption.

\section{A more precise estimation for the interfacial tension}

In order to understand the role of model parameters and apply the model for real systems, more sophisticated analytical calculation for the interfacial tension is needed. First, we approximate the equilibrium planar surfactant profile by taking into account the algebraic Euler-Lagrange equation Eq. (7) instead of the constant field approximation. Then, we present calculations for the intercial tension in our proposed model $\lambda_{1}=d$ and $\lambda_{2}=a+d$ in both the symmetric $(e=0)$ and the general asymmetric $(e \neq 0)$ case.

\section{Symmetric case.}

As discussed above, now we take into account that the equilibrium planar surfactant profile $\psi^{*}(x)$ varies in space. Since Eq. (7) cannot be solved analytically for $c \neq 0$, first we assume that $\psi^{*}(x)$ remains sufficiently close to $\psi_{0}$ to use the second-order Taylor expansion of the logarithmic term in $\mathcal{F}_{\psi}$ around $\psi_{0}$. Then 
we expand Eq. (7) for $\phi^{*}(x)$ defined by Eq. (15) and $\psi^{*}(x)=\psi_{0}+\delta \psi^{*}(x)$ with respect to $\delta \psi^{*}(x)$ up to the linear order, yielding

$$
\delta \psi^{*}(x) \approx \frac{\Delta \hat{f}\left[\phi^{*}(x)\right]}{c-\frac{1}{\beta \psi_{0}\left(1-\psi_{0}\right)}},
$$

where $\Delta \hat{f}\left[\phi^{*}(x)\right]$ is defined by Eq. (20) with $\phi^{-}=-\phi_{0}$. Since there are two leading terms in $\Delta f\left[\phi^{*}(x)\right]$, namely $g\left[\phi^{*}(x)\right] \propto \operatorname{sech}^{4}(x / 2)$ and $\left[\partial_{x} \phi^{*}(x)\right]^{2} \propto \operatorname{sech}^{4}(x / 2)$, $\psi^{*}(x)$ is simply approximated as

$$
\delta \psi^{*}(x) \approx A \operatorname{sech}^{4}(x / 2),
$$

where the amplitude $A$ can be calculated by taking Eqns. (26) and (27) at $x=0$, yielding

$$
A=\frac{\theta\left(\psi_{0}\right)}{c-\frac{1}{\beta \psi_{0}\left(1-\psi_{0}\right)}},
$$

where $\theta\left(\psi_{0}\right)$ is defined by Eq. (22). In the symmetric case $\mu_{\psi}=0$, therefore, the interfacial tension simply reads as

$$
\sigma=\int_{-\infty}^{+\infty} d x\left\{\mathcal{F}\left[\phi^{*}(x), \psi^{*}(x)\right]-\mathcal{F}_{0}-\mu_{\psi}\left[\psi^{*}(x)-\psi_{0}\right]\right\}
$$

Using Eqns. (15) and (27), and taking into account the Taylor expansion of the logarithmic term in $\mathcal{F}_{\psi}$ up to the second order, the interfacial tension reads as

$$
\sigma=\sigma_{C H}+\sigma_{\psi}+\sigma_{1}+\sigma_{e x},
$$

where

$$
\begin{aligned}
\sigma_{C H}= & \sigma_{0}\left[\phi_{0}^{2}\left(2-\phi_{0}^{2}\right)\right] \\
\sigma_{\psi}= & \frac{32}{35} A^{2}\left[\frac{1}{\beta \psi_{0}\left(1-\psi_{0}\right)}-c\right] \\
\sigma_{1}= & -\frac{2}{105} \phi_{0}^{2}\left[8 A\left(3 a+10 d-4 d \phi_{0}^{2}\right)\right. \\
& \left.\quad+35\left(a+4 d-2 d \phi_{0}^{2}\right) \psi_{0}\right] \\
\sigma_{e x}= & -\frac{2}{15} a \phi_{0}^{2}\left(8 A+15 \psi_{0}\right),
\end{aligned}
$$

where $\phi_{0}$ and $A$ are defined by Eqns. (12) and (28), respectively. The first correction to the constant surfactant field approximation around $\psi_{0}=0$ comes from the Taylor expansion of Eq. (29):

$$
\kappa=1-(2 a+d+q) \psi_{0}+O\left(\psi_{0}^{2}\right),
$$

where $q=\left[\left(33 a^{2}+40 a d+12 d^{2}\right) / 70\right] \beta$, accounting for the correction due to $\psi^{*}(x) \neq \psi_{0}$. Note that Eq. (18) reads as $\kappa=1-(2 a+d) \psi_{0}+O\left(\psi_{0}^{2}\right)$ for $\lambda_{1}=d$ and $\lambda_{2}=a+d$, i.e. Eq. (34) starts with a different slope at $\psi_{0}=0$.

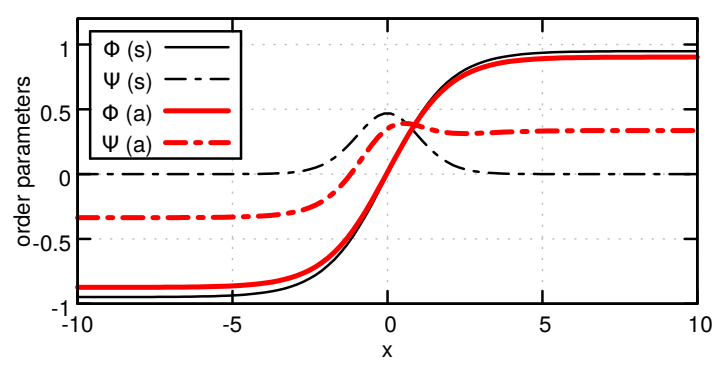

FIG. 3. Equilibrium planar interfaces as predicted by Eqns. $(15)$ and (27) in the symmetric case $(e=0$, solid and dashed red curves) case, and with the corrections described by Eqns. (41) and (42) for the asymmetric case $(e=8$, solid and dashed red curves), respectively, at parameters $\beta^{-1}=100, d=10$, $a=1$ and $\psi_{0}=0.05$. The surfactant interfaces are normalized as $\Psi(x)=\beta^{-1}\left[\psi^{*}(x)-\psi_{0}\right]$.

\section{Asymmetric case.}

In case of $e \neq 0$ Eq. (8) is not symmetric, yielding the general bulk equilibrium solution $\phi^{+} \neq \phi^{-}$and $\psi^{-} \neq$ $\psi^{+}$. Therefore, one has to consider the full equilibrium problem:

$$
\begin{aligned}
& \mu_{\phi}^{+}=\mu_{\phi}^{-} \\
& \mu_{\psi}^{+}=\mu_{\psi}^{-} \\
& \omega^{+}=\omega^{-},
\end{aligned}
$$

where $\omega=\mathcal{F}-\mu_{\phi}^{ \pm} \phi-\mu_{\psi}^{ \pm} \psi$ is the grand potential density, whereas $\mu_{\phi}^{ \pm}=\left.\mu_{\phi}\right|_{\phi^{ \pm}, \psi^{ \pm}}, \mu_{\psi}^{ \pm}=\left.\mu_{\psi}\right|_{\phi^{ \pm}, \psi^{ \pm}}$, and $\omega^{ \pm}=$ $\left.\omega\right|_{\phi^{ \pm}, \psi^{ \pm}}$. Note that there are 4 variables $\left(\phi^{ \pm}\right.$and $\left.\psi^{ \pm}\right)$ and 3 equations. Since we're interested in the physical quantities as a function of the surfactant load $\psi_{0}$, we introduce

$$
\phi^{ \pm}= \pm \phi_{0} \pm \delta \phi_{ \pm} \quad \text { and } \quad \psi^{ \pm}=\psi_{0} \pm \delta \psi_{0},
$$

where $\phi_{0}$ is defined by Eq. (15) and $\psi_{0}$ is the only free parameter, the "average" surfactant load $\psi_{0}=\left(\psi^{-}+\right.$ $\left.\psi^{+}\right) / 2$. (Note that this is not the real average, since the extra amount of the surfactant at the interface is not considered here.) This variable transformation is convenient, since the equilibrium values reduce to the symmetric solution for $e=0$. Assuming that $e$ and $c$ are chosen so that $\left|\delta \psi_{0} / \psi_{0}\right|$ and $\left|\delta \phi_{ \pm} / \phi_{0}\right|$ are sufficiently small, Eqns. (35)-(37) can be expanded up to linear order in $\delta \psi_{0}$ and $\delta \phi_{ \pm}$around the symmetric solution, resulting in

$$
\begin{aligned}
\delta \psi_{0} & =\frac{e \phi_{0}}{\mu_{\psi \psi}-\mu_{\phi \phi}^{-1}\left[e^{2}+\left(\mu_{\phi \psi}^{0}\right)^{2}\right]} \\
\delta \phi_{ \pm} & =\frac{e \mp \mu_{\phi \psi}^{0}}{\mu_{\phi \phi}} \delta \psi_{0},
\end{aligned}
$$

where $\mu_{\phi \phi}=\left.\left(\partial^{2} \mathcal{F} / \partial \phi^{2}\right)\right|_{\phi_{0}, \psi_{0}}, \mu_{\psi \psi}=\left.\left(\partial^{2} \mathcal{F} / \partial \psi^{2}\right)\right|_{\phi_{0}, \psi_{0}}$, and $\mu_{\phi \psi}^{0}=\left.\left(\partial^{2} \mathcal{F} / \partial \phi \partial \psi\right)\right|_{\phi_{0}, \psi_{0}} ^{e=0}$. The equilibrium planar 
interfaces can be written as

$$
\begin{aligned}
& \phi_{a}^{*}(x) \approx \phi^{*}(x)+\delta \phi_{a}^{*}(x) \\
& \psi_{a}^{*}(x) \approx \psi^{*}(x)+\delta \psi_{a}^{*}(x),
\end{aligned}
$$

where the corrections are defined as

$$
\begin{aligned}
\delta \phi_{a}^{*}(x) & =\delta \phi_{+} \frac{\tanh \left(\frac{x}{2}\right)+1}{2}+\delta \phi_{-} \frac{\tanh \left(\frac{x}{2}\right)-1}{2} \\
\delta \psi_{a}^{*}(x) & =\delta \psi_{0} \tanh \left(\frac{x}{2}\right)+(B-A) \operatorname{sech}^{4}(x / 2)
\end{aligned}
$$

(see Fig 3). Here $B$ is determined at $x=0$, where $\phi_{a}^{*}(0)=\left(\phi^{-}+\phi^{+}\right) / 2$ and $\psi_{a}^{*}(0)=\psi_{0}+B$. Expanding the Euler-Lagrange equation described by Eq. (7) up to the first order around $\psi_{0}$ results in

$$
B=\frac{\Delta \hat{f}\left[\phi_{a}^{*}(x)\right]_{x=0}}{c-\frac{1}{\beta \psi_{0}\left(1-\psi_{0}\right)}}-\delta \psi_{0}
$$

which reduces to Eq. (28) for $e=0$. When calculating the interfacial tension, one has to take into account that Eqns. (35)-(37) have been taken up to the first order in calculating equilibrium, the correction to the interfacial tension must be calculated accordingly. The interfacial tension is calculated as

$$
\sigma=\int_{-\infty}^{+\infty} d x\left\{\omega-\omega^{-}\right\}
$$

where thermodynamic grand potential density has been expanded in Eq. (37) around the symmetric solution $\phi^{*}(x)$ and $\psi^{*}(x)$ up to the linear order as

$$
\begin{aligned}
\omega \approx & \mathcal{F}^{(1)}-\mu_{\phi}^{ \pm} \phi^{*}(x)-\mu_{\psi}^{ \pm} \psi^{*}(x) \\
& -\mu_{\phi, 0}^{ \pm} \delta \phi^{*}(x)-\mu_{\psi, 0}^{ \pm} \delta \psi^{*}(x)
\end{aligned}
$$

where $\mu_{\phi}^{ \pm}$and $\mu_{\psi}^{ \pm}$are taken up to the first order in $\delta \phi^{ \pm}$ and $\delta \psi^{ \pm}$around the symmetric solutions $\mu_{\phi, 0}^{ \pm}$and $\mu_{\psi, 0}^{ \pm}$, respectively (the same applies for $\mathcal{F}^{(1)}$, i.e. it is taken around $\mathcal{F}_{0}$ up to the first order with respect to $\delta \phi_{a}^{*}(x)$ and $\delta \psi_{a}^{*}(x)$, and their spatial derivatives). Note, that although the terms containing $\mu_{\phi, 0}^{ \pm}$and $\mu_{\psi, 0}^{ \pm}$cancel in the bulk phases, $\mu_{\psi, 0}^{-} \neq \mu_{\psi, 0}^{+}$and $\mu_{\phi, 0}^{-} \neq \mu_{\phi, 0}^{+}$in general, because of the presence of the asymmetric term. Therefore, Eq. (45) is not univalent at the interface. Apparently this was the price of using linear approximation for both the background potentials and the grand potential density. To resolve this discrepancy, we introduce the "average" grand potential density instead of Eq. (45) as:

$$
\begin{aligned}
\bar{\omega}:= & \mathcal{F}^{(1)}-\bar{\mu}_{\phi} \phi^{*}(x)-\bar{\mu}_{\psi} \psi^{*}(x) \\
& -\bar{\mu}_{\phi, 0} \delta \phi^{*}(x)-\bar{\mu}_{\psi, 0} \delta \psi^{*}(x),
\end{aligned}
$$

where $\bar{\mu}_{\phi}=\left(\mu_{\phi}^{+}+\mu_{\phi}^{-}\right) / 2$ and $\quad \bar{\mu}_{\psi}=\left(\mu_{\psi}^{+}+\mu_{\psi}^{-}\right) / 2$. Note that Eq. (46) is univalent, and $\bar{\mu}_{\psi}^{+}=\bar{\mu}_{\psi}^{-}$, and it is also easy to show that it results in the equilibrium condition identical to Eq. (37), i.e. $\omega^{+}=\omega^{-} \Leftrightarrow \bar{\omega}^{+}=\bar{\omega}^{-}$. After some trivial algebraic manipulation and integration the interfacial tension reads as

$$
\sigma=\sigma_{e=0}+\sigma_{\delta g}+\sigma_{\psi}+\sigma_{\delta \phi}+\sigma_{\delta \psi}
$$

where $\sigma_{e=0}$ is the interfacial tension in the symmetric case defined by Eq. (29), while the corrections read as:

$$
\begin{aligned}
\sigma_{\delta g}= & \frac{4 \phi_{0}}{105}\left\{12(A-B)(a+d) \phi_{0}\right. \\
& \left.-\Delta \phi_{+}\left[24 A(a+d)-35\left(1-\{a+d\} \psi_{0}\right)\right]\right\} \\
\sigma_{\psi}= & -2 e \delta \psi_{0}\left(\mu_{\phi \psi}^{0} / \mu_{\phi \phi}\right) \\
\sigma_{\delta \phi}= & \frac{4}{105} \phi_{0} \Delta \phi_{+}\left\{7\left[15-2 a A+2 A d+15(a-d) \psi_{0}\right]-\right. \\
- & \left.2 \phi_{0}^{2}\left(70+3 A d-70 d \psi_{0}\right)\right\}+\frac{8}{3} A e \Delta \phi_{-} \\
\sigma_{\delta \psi}= & \frac{192 A(A-B)}{105 \beta \psi_{0}\left(\psi_{0}-1\right)}+\frac{4}{105}\{48 A(A-B) c \\
+ & \left.\phi_{0}\left[105 e \delta \psi_{0}+4(A-B) \phi_{0}\left(7\{a+d\}-4 d \phi_{0}^{2}\right)\right]\right\},
\end{aligned}
$$

where $\Delta \phi_{ \pm}=\left[\left(\delta \phi_{-}\right) \pm\left(\delta \phi_{+}\right)\right] / 2$. It is trivial that $A-B \propto$ $e$, meaning that the correction terms are proportional to $e$, i.e. they all vanish for $e \rightarrow 0$. The relative interface tension reads as

$\kappa=1-(2 a+d+q) \psi_{0}+\left(p+\frac{2 a^{3} \beta^{2}}{35} e\right) \psi_{0}^{2}+O\left(\psi_{0}^{3}, e^{2}\right)$,

where $p=a^{2}+(\beta / 70)\left\{66 a^{3}+8 a d(5+d-5 c \beta)-\right.$ $\left.12 d^{2}(c \beta-1)+3 a^{2}[11(1-c \beta)+18 d]\right\}$ and $q$ is defined after Eq. (34). Therefore, the asymmetry has only marginal effect on the interfacial tension near $\psi_{0}=0$, however, the behavior of the interfacial tension near the critical point necessitates further analysis.

\section{Analysis near the critical point}

While the interfacial tension and the interface width for the surfactant-free system are natural units of the theory, the critical point $\psi_{c}$ is defined by exclusively the properties of the emulsifier via Eq. (13). Substituting $\psi_{0}=\psi_{c}$ into Eqns. (29) and (47) also yields $\kappa=0$ (for $a \neq 0$ ), therefore, the critical point is independent from the values of $\beta, c$ and $e$, showing the robustness of the theory. In the symmetric case the relative interfacial tension near the critical point can be obtained by expanding Eq. (29) around $\psi_{c}$ for $\delta \bar{\psi}=\psi_{0}-\psi_{c} \leq 0$, resulting in

$$
\kappa=\left[\frac{33 \alpha+70 a \psi_{c}^{2}\left(1+c \psi_{c} \alpha\right)}{70 a^{2} \psi_{c}^{5}\left(1+c \psi_{c} \alpha\right)}\right] \delta \bar{\psi}^{2}+O\left(\delta \bar{\psi}^{3}\right),
$$

where $\alpha=\beta\left(\psi_{c}-1\right)$. Comparing Eq. (49) to the results of previous works for the interfacial tension lowering [defined as $\Delta \sigma=(\kappa-1) \sigma_{0}$ ] indicates that the previously suggested $\Delta \sigma \propto \log \left(1-k \psi_{0}\right)$ relationship is not true for $\psi_{0} \lesssim \psi_{c} \ll 1$. This is not surprising, taking into account that $\Delta \sigma \propto \log \left(1-k \psi_{0}\right)$ emerges from the 


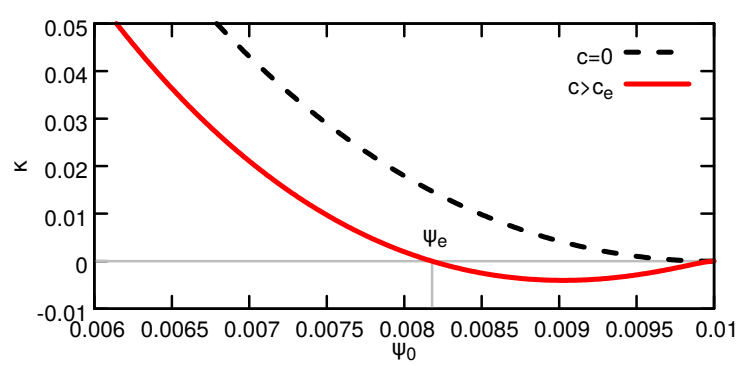

FIG. 4. Relative interfacial tension $\kappa$ as a function of the surfactant load $\psi_{0}$, as predicted by Eq. (29) for $\beta^{-1}=120$, $a=201$ and $d=-101$. Note that in case of $c=1.2 \times 10^{4}>$ $c_{e}=9775.8$ an emulaification point $\psi_{e}=0.00818$ appears, above which spontaneous emulsification occurs due to the negative interfacial tension.

Langmuir isotherm described by Eq. (23). Accordingly, in the Work of van der Sman and van der Graaf [14] the logarithmic expression has been found valid even for $\psi_{0}=0 \ldots 0.7$, due to the fact that $\psi_{c}=2$ applied in that case, and the Langmuir isotherm was valid on almost the entire range $\psi_{0} \in[0,1]$. In contrast, in the work of Liu and Zhang [17] the $\log \left(1-k \psi_{0}\right)$ fit clearly shows strong error at significant surfactant loads, where the Langmuir isotherm is not valid anymore (this is typical for $\psi_{c} \approx 1$ ).

Eq. (49) plays an important role also from the viewpoint of spontaneous emulsification: If the coefficient of $\delta \bar{\psi}^{2}$ is non-negative, spontaneous emulsification cannot happen at $\psi_{0}<\psi_{c}$, since the interfacial tension is nonnegative in the entire range. In contrast, one can achieve negative interfacial tensions for $\psi_{0}<\psi_{c}$ when

$$
c>c_{e}=-\frac{33}{70 a \psi_{c}^{3}}+\frac{\beta^{-1}}{\psi_{c}\left(1-\psi_{c}\right)}
$$

applies (or, in other words, when the interaction between adjacent surfactant layers is strong enough). In these cases an emulsification point $\left(\psi_{e}<\psi_{c}\right)$ appears (see Fig 4 ), at which the interfacial tension vanishes with finite $\phi_{0}$. In addition, above $\psi_{e}$ emulsification starts spontaneously due to hydrodynamic instabilities emerging from the negative interfacial tension.

It is important to mention, that the analysis is not straightforward in the asymmetric case. Although both $\delta \phi_{ \pm} \rightarrow 0$ and $\phi_{0} \rightarrow 0$ apply for $\psi_{0} \rightarrow \psi_{c}$, Eq. (40) results in $\delta \phi_{ \pm} / \phi_{0}=-1+O(\sqrt{\delta \bar{\psi}})$ (where $\delta \bar{\psi}=\psi_{c}-\psi_{0} \geq 0$ ), while $\delta \phi_{ \pm} / \phi_{0} \equiv 0$ for $e=0$. This indicates a qualitatively different behavior of the interfacial tension near the critical point in case of $e=0$ and $e \neq 0$ : Although the interfacial tension vanishes for $\psi_{0}=\psi_{c}$ exactly for any $e \in \mathbb{R}$, Eq. (47) may not converge for $\psi_{0} \rightarrow \psi_{c}$ in case of $e \neq 0$. This discrepancy emerges from the linear approximation of Eqns. (35)-(37), however, a more accurate derivation is out of the scope of the present work.

\section{NUMERICAL SIMULATIONS}

\section{A. Methodology}

\section{Numerical solution of the Euler-Lagrange equations}

In order to validate Eq. (29), we determine the interfacial free energy using the numerical solution of the EulerLagrange equations described by Eqns. (6) and (7) as follows. Eq. (6) is a second-order differential equation for $\phi$, necessitating 2 boundary conditions to define a two-point boundary problem, while Eq. (7) is an algebraic equation prescribing the relationship between $\phi$ and $\psi$ implicitly for a given $\psi_{0}$. The boundary conditions for the planar interface solution, however, read as $\phi(x \rightarrow \pm \infty) \rightarrow \phi^{ \pm}$, $\partial_{x} \phi(x \rightarrow \pm \infty) \rightarrow 0$, representing 4 constraints. Therefore, the problem is overdetermined, and in these cases shooting-type numerical integrators are not suitable in principle [22]. In addition, the problem is ill-posed in a sense that it is translational invariant, namely, if $\phi^{*}(x)$ and $\psi^{*}(x)$ is a solution, then $\phi^{*}\left(x-x_{0}\right)$ and $\psi^{*}\left(x-x_{0}\right)$ is also solution for any $x_{0} \in \mathbb{R}$. Consequently, relaxationtype methods might also fail to converge on a finite range [22]. In order to avoid this problem, first we transform the equilibrium planar interface problem from an infiniterange problem into a finite-range problem by introducing the new independent coordinate:

$$
\hat{x}:=\tanh (x / 2) .
$$

The new variables then read as $\hat{\phi}(\hat{x})=\{\phi[x(\hat{x})]-\Delta\} / h$, where $\Delta=-\left(\phi^{-}+\phi^{+}\right) / 2$ and $h=\left(\phi^{+}-\phi^{-}\right) / 2$, yielding $\hat{\phi}( \pm 1)= \pm 1$, and $\hat{\psi}(\hat{x})=\psi[x(\hat{x})]$. For the conversion, however, the values $\phi^{ \pm}$must be known, but these are known only for $e=0$ analytically, therefore, we will consider only the symmetric case hereafter, where $\hat{\phi}(\hat{x})=\phi[x(\hat{x})] / \phi_{0}$. The spatial derivatives can be then expressed as:

$$
\begin{aligned}
& \partial_{x} \tilde{\phi}=t \partial_{\hat{x}} \hat{\phi} \\
& \partial_{x}^{2} \tilde{\phi}=t\left[t\left(\partial_{\hat{x}}^{2} \hat{\phi}\right)+\hat{x}\left(\partial_{\hat{x}} \hat{\phi}\right)\right],
\end{aligned}
$$

where $\tilde{\phi}=\phi / \phi_{0}$ and $t=1 /\left(\partial_{\hat{x}} x\right)=\left(1-\hat{x}^{2}\right) / 2$. The Euler-Lagrange equations can be trivially transformed by using Eqns. (52) and (53) and solved with the boundary conditions $\hat{\phi}( \pm 1)= \pm 1$ and $\psi( \pm 1)=\psi_{0}$ with a relaxation method [22]. After having the solution $\hat{\phi}^{*}(\hat{x})$ and $\hat{\psi}^{*}(\hat{x})$ one can calculate the interfacial tension as

$$
\begin{aligned}
& \sigma=\int_{-1}^{+1} d \hat{x}\left\{t \left(\mathcal{F}\left[\hat{\phi}^{*}(\hat{x}), \hat{\psi}^{*}(\hat{x})\right]-\mathcal{F}_{0}\right.\right. \\
&\left.\left.-\tilde{\mu}_{\psi}\left[\hat{\psi}^{*}(\hat{x})-\psi_{0}\right]\right)\right\}
\end{aligned}
$$

where the integrand contains the transformed derivative $\left[\partial_{x} \phi^{*}(x)\right]^{2}=\left[t \phi_{0} \partial_{\hat{x}} \hat{\phi}^{*}(\hat{x})\right]^{2}$, naturally. 


\section{Numerical solution of the dynamic equations}

Eqns. (9) and (10) represent a fourth order nonlinear parabolic PDE system. We use an advanced operatorsplitting based semi-implicit pseudo-spectral method developed by Tegze et al [23] to solve the dynamic equations numerically with periodic boundary conditions. With this method large time steps are allowed to be used, contrary to a finite difference method, where the stability criterion limits the time steps as a $h^{4}$, where $h$ is the spatial discretization step.

\section{B. Parameters}

For the validation of the model / numerical simulations we determine the model parameters for a model system mimicking water $/ \mathrm{CO}_{2} /$ macromolecular surfactant systems. Thus, the interfacial tension is in the order of $50 \mathrm{~mJ} / \mathrm{m}^{2}$ [24], and the width of the interface loaded by macromolecules is in the order of $\delta_{0} \approx 0.1 \mu \mathrm{m}$ [25]. The molecular weight of macromolecular surfactants is typically in the order of $1000 \mathrm{~g} / \mathrm{mol}$, while the density is considered to be approximately $1 \mathrm{~g} / \mathrm{cm}^{3}[26,27]$, yielding the average density of the system $\rho \approx 1000 \mathrm{~kg} / \mathrm{m}^{3}$. We choose the critical point $\psi_{c} \approx 0.01$ [27], therefore, the average molar volume of the system can be approximated as $v_{0}=50 \mathrm{~cm}^{3} / \mathrm{mol}$. The liquid-liquid diffusion coefficient is typically $D_{0}=5 \times 10^{-9} \mathrm{~m}^{2} / \mathrm{s}$ [28] and for the sake of simplicity $\tilde{M}(\phi)=1$ is chosen. The typical diffusion coefficient of macromolecules in water reads as $D_{\psi} \approx 5 \times 10^{-11} \mathrm{~m}^{2} / \mathrm{s}$ at room temperature $(T \approx 300 \mathrm{~K})$ [29]. Considering these physical parameters yields the model parameters

$$
\beta^{-1}=120 \quad \text { and } \quad \tilde{\tau}_{\psi}=3600 .
$$

Furthermore, we choose

$$
a=201, \quad d=-101 \text { and } c=0,
$$

yielding a realistic $\kappa$ curve with $\psi_{c}=0.01$ [27]. Now one can see the huge difference between the cases considered previoulsy and in this work by comparing Figs 3 and 4 of Sagisaka et al. [27] to Fig 3 of Liu and Zhang [17]. In the water/liquid $\mathrm{CO}_{2} /$ macromolecular surfactant system the interfacial tension drops suddenly for small surfactant loads $\left(\psi_{0} \ll \psi_{c} \approx 0.01\right)$, then it converges to zero for $\psi_{0} \rightarrow \psi_{c}$. In contrast, the relative interfacial tension lowering behaves qualitatively different in the work of Liu and Zhang, prescribing slow changes for $\psi_{0} \ll 1$ and fast changes for $\psi_{0} \rightarrow 1$ (together with $\left.\psi_{c}>1\right)$.

The Euler-Lagrange equations were solved for $e=0$ as a function of the surfactant load to test the validity of our approximations for the interfacial tension, while the time evolution of the system was studied as a function of the surfactant load in 2 dimensions on a $1024 \times 1024$ grid with $\Delta x=\Delta y=\Delta t=1$. The initial conditions were $\phi(\mathbf{r}, 0)=\phi_{0}(\Phi+\alpha \mathcal{R}[-1,1])$ and $\psi(\mathbf{r}, 0)=\psi_{0}$, where $\Phi \in[-1: 1]$ is a fixed volume fraction, $\mathcal{R}[-1,1]$ is a random noise of uniform distribution on $[-1,1]$, and $\alpha \ll 1$.

\section{Results}

\section{Interfacial tension}

First the validity of our analytic approximations for the interfacial tension is examined. Fig. 5 shows the relative interfacial tension obtained from different approaches in case of $e=0$. Although the constant surfactant field approximation gives a reasonable estimation, Eq. (29) matches the numerical results almost perfectly. Since all curves converge to 0 in case of $\psi_{0} \rightarrow \psi_{c}$, it is worth to investigate the realtive errors too. Both the constant surfactant approximation (compared to the more precise analytic approximation) and the analytic calculation (compared to the numerical results) show finite relative error at the critical point, which means that $\sigma=a\left(\psi_{c}-\psi_{0}\right)^{2}+O\left[\left(\psi_{c}-\psi_{0}\right)^{3}\right]$ applies for all curves, but with different 'a' coefficients. We also note, that the relative error between the precise analytic approximation and the numeric results shows a maximum at $\psi_{0} \approx 0.0002$, which is due to the fact that the error increases with increasing deviation of the surfactant profiles obtained from the different methods. Since the surfactant profile is exactly zero at $\psi_{0}=0$, and the interfacial tension vanishes at $\psi_{0}=\psi_{c}$ (the solution is analytic in both cases), the interfacial tensions coincide at these points, regardless of the method we choose. Consequently, $\left|\sigma_{1}-\sigma_{2}\right|=0$ applies at $\psi_{0}=0$ and $\psi_{0}=\psi_{c}$, but otherwise the error is finite in between, showing a maximum on $\psi_{0} \in\left(0, \psi_{c}\right)$. The relative error slightly modifies this picture, since it can be finite at the critical point because of the reasons described above. Summarizing, the relative errors indicate that the model parameters should be fitted via Eq. (49) near the critical point rather than using Eq. (18), because the coefficient for the precise analytic estimation containing additional factors compared to (49) is in a qualitatively better agreement with the numerical results. The importance of Eq. (29) is then two-fold: First, the model can be calibrated for real systems analytically. Second, it shows that the critical point does not change as a function of the level of precision in determining the interfacial tension, showing the robustness of the theory.

\section{Phase separation}

a. Symmetric case. The time evolution of the system was scanned as a function of $\psi_{0}$. Figs 6 and 7 show snapshots of the simulations at $t=100,200,500$ and 1000 (corresponding to panels a-d) in case of $\psi_{0}=0.005$ and 

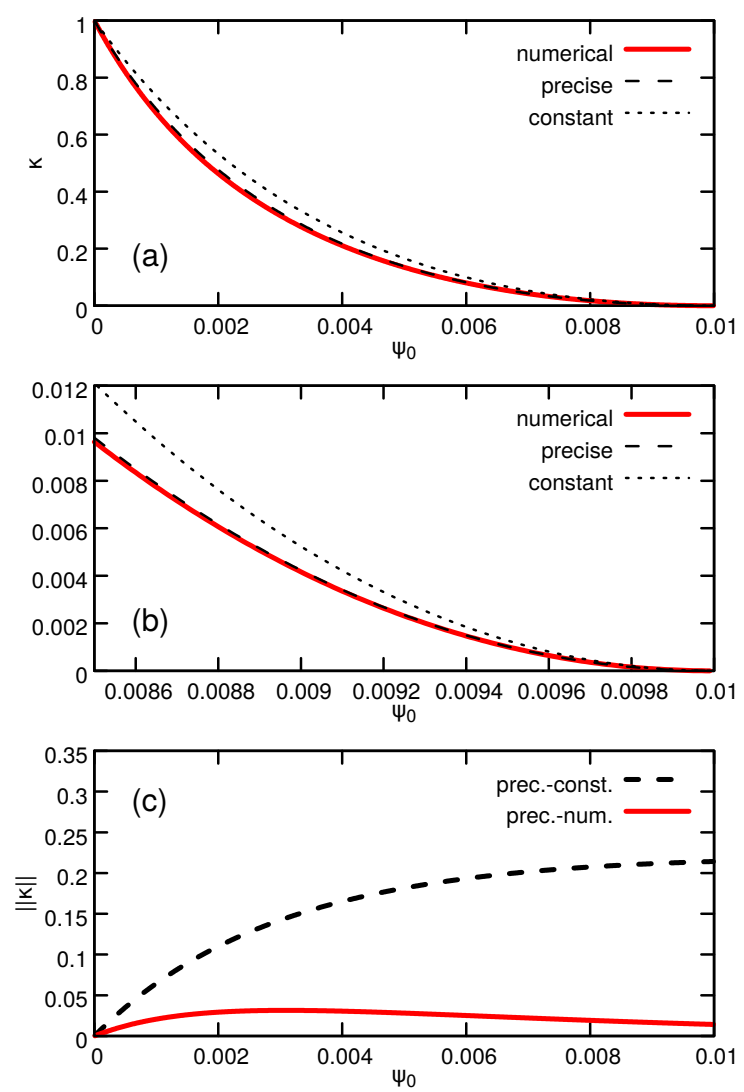

FIG. 5. Panel (a): Dynamic factor and relative interfacial tension predicted by different approaches (numerical solution of the Euler-Lagrange equations, precise analytical calculation and constant surfactant field approximation) as a function of the surfactant load. Panel (b): Magnification of panel a near the critical point $\psi_{c}=0.01$. Panel (c): Relative deviation $\|\kappa\|=2\left|\left(\sigma_{1}-\sigma_{2}\right) /\left(\sigma_{1}+\sigma_{2}\right)\right|$, where $\sigma_{1}$ and $\sigma_{2}$ are the interfacial tensions from the precise calculation and the constant surfactant approximation (dashed curve), and from the precise calculation and the numerical solution of the EulerLagrange equations (solid curve), respectively.

$\psi_{0}=0.0099$, respectively. It can be clearly seen that the phase separation is significantly slower for $\psi_{0}=0.0099$ (Fig 7) than for $\psi_{0}=0.005$ (Fig 6). To quantify the results, we introduce the amount of liquid-liquid interfaces:

$$
Q(t):=\int d V\left\{[\nabla \hat{\phi}(\mathbf{r}, t)]^{2}\right\}
$$

where $\hat{\phi}(\mathbf{r}, t)=\phi(\mathbf{r}, t) / \phi_{0} \in[-1,1]$ is the normalized liquid-liquid order parameter. Fig 8.a shows $Q(t)$ for different surfactant loads $\left(\psi_{0} / \psi_{c}=\right.$ $0.0,0.25,0.5,0.75,0.95,0.99$ and 0.999 from bottom to top, respectively). The curves are linear and parallel to each other on the log-log plot, implying the master curve

$$
Q(t)=\left[t / \tau\left(\psi_{0}\right)\right]^{q}
$$

where the time scale reads as $\tau\left(\psi_{0}\right)=\exp \left[p\left(\psi_{0}\right) / q\right]$, while $p\left(\psi_{0}\right)$ and $q$ are the parameters of the linear fit
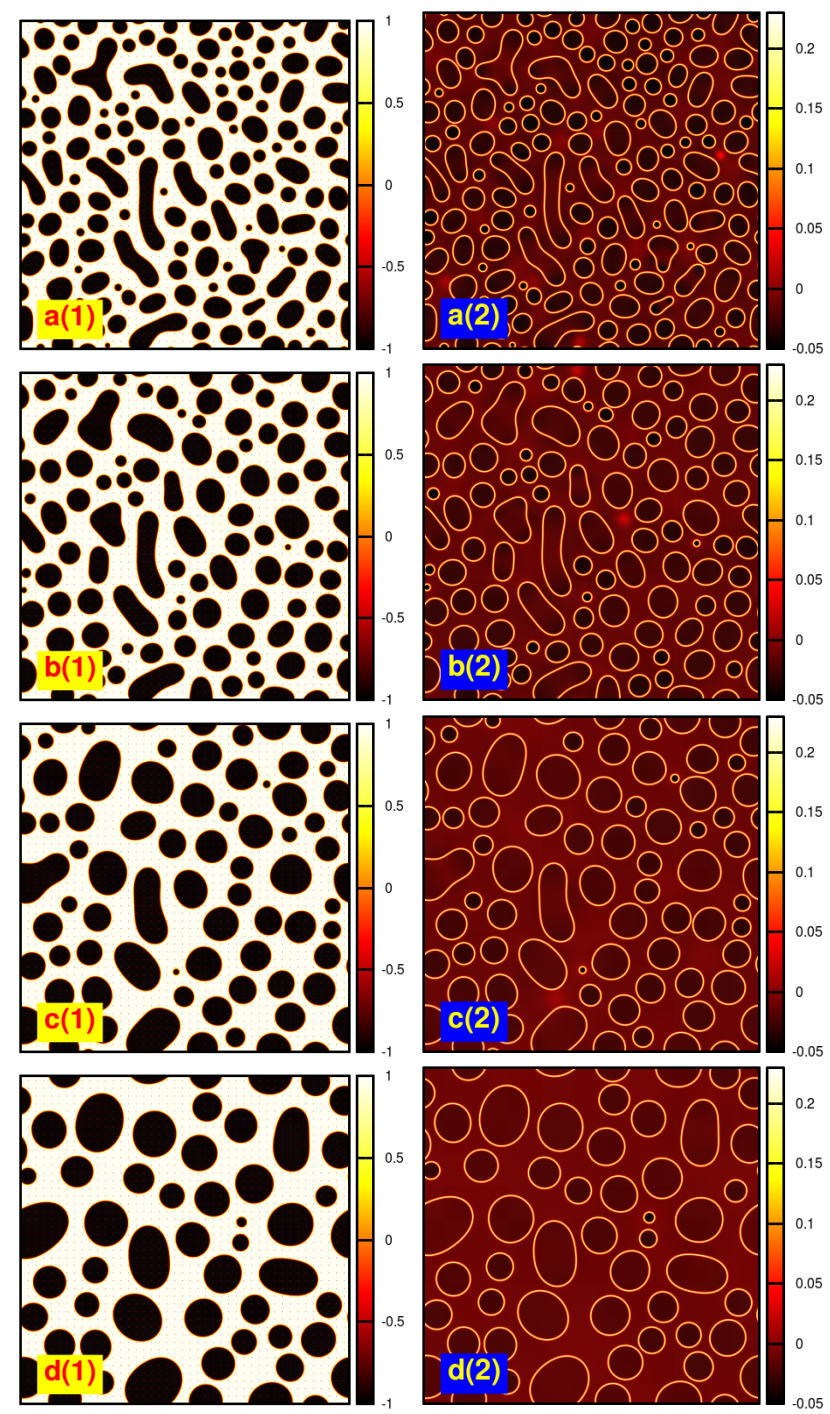

FIG. 6. Time evoltion of the system for average surfactant load $\psi_{0}=0.005$ in case of $e=0 . \phi(\mathbf{r}, t) / \phi_{0}$ and $\left[\psi(\mathbf{r}, t) / \psi_{0}\right]-1$ are shown on panels $\mathrm{a}(1)-\mathrm{d}(1)$ and $\mathrm{a}(2)-\mathrm{d}(2)$, respectively at $t=100,200,500$ and 1000 (time passes from top to bottom).

$\log [Q(t)]=p\left(\psi_{0}\right)-q \log (t)$. Eq. (56) means that the qualitative behavior of the system is independent from the surfactant load, therefore, the dynamic factor can be written as

$$
s=\frac{\tau(0)}{\tau\left(\psi_{0}\right)}=\exp \left[-\Delta p\left(\psi_{0}\right) / q\right],
$$

where $\Delta p\left(\psi_{0}\right)=p\left(\psi_{0}\right)-p(0)$, i.e. the vertical distance between the lines corresponding to $\psi_{0}$ and $\psi_{0}=0$ in Fig 8.a. The numerical simulations resulted in $q \approx 0.28$, while the distances read as $\Delta p\left(\psi_{0}\right)=0.0,0.22822,0.45237,0.70846,1.08054,1.50933$ and 2.16971 (from bottom to top, respectively). The calculated dynamic factors are shown in Fig 8.b, as a function of the surfactant load. It is obvious that the 

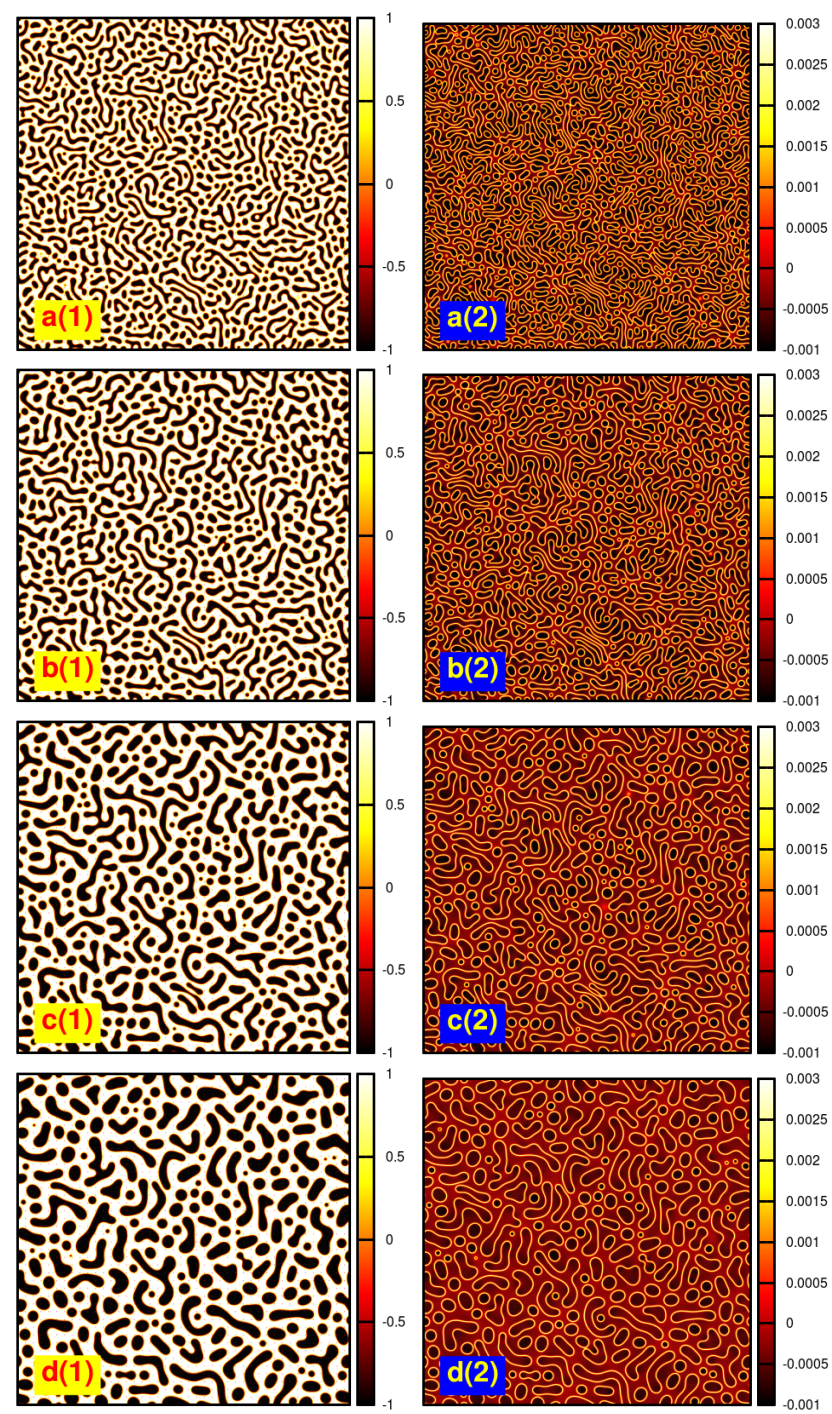

FIG. 7. Time evolution of the system for average surfactant load $\psi_{0}=0.0099$ in case of $e=0 . \phi(\mathbf{r}, t) / \phi_{0}$ and $\left[\psi(\mathbf{r}, t) / \psi_{0}\right]-$ 1 are shown on panels a $(1)-d(1)$ and a $(2)-d(2)$, respectively at $t=100,200,500$ and 1000 (time passes from top to bottom).

numerical dynamic factor (i.e. the relative speed of phase separation) follows the reduction of the interfacial tension, rather than Eq. (16), or, in other words, the dynamical system is driven by the interfacial tension. It is an important result, showing that even though the constant surfactant approximation results in a reliable estimation for the relative interfacial tension, it is absolutely not sufficient to predict the speed of phase separation. The error of $s$ relative to $\kappa$ is due to (i) finite size effects, and (ii) the fact that the surfactant load in the bulk phases changes constantly even during a single simulation because of the conservative dynamics (however, this change is less than $1 \%$ in our simulations).
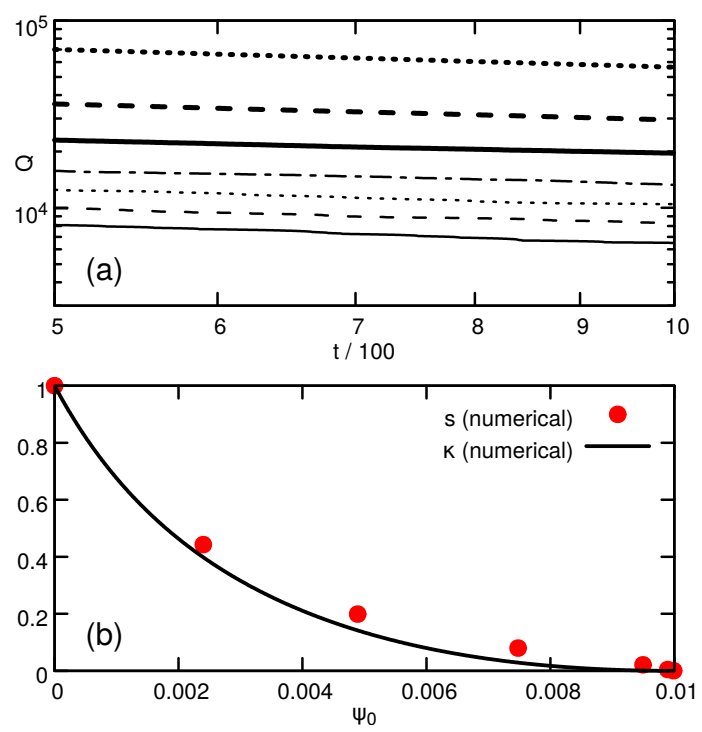

FIG. 8. Time evolution of the symmetric system: (a) Amount of liquid-liquid interfaces as a function of time for average surfactant loads $\psi_{0} / \psi_{c}=0.0,0.25,0.5,0.75,0.95,0.99$ and 0.999 (from bottom to top, respectively). (b) Dynamic factor (or the relative speed of phase separation) defined by Eq. (57) (red dots) at surfactant loads corresponding to panel a, compared to the relative interfacial tension $\kappa$ obtained from the numerical solution of the Euler-Lagrange equations.

b. Asymmetric case. Finally, the effect of the asymmetry is investigated. We apply $e=10$, yielding the estimated relative difference $\left|\delta \psi_{0} / \psi_{0}\right| \leq 10 \%$, indicating significant asymmetry, as also shown by Figs 9 and 10. Note, however, that Fig 10 corresponds to $\psi_{0}=0.0097$ now, indicating a slight shift in the critical point due to the asymmetry. Furthermore, the amplitude of the surfactant at the interface vanishes relative to $\delta \psi_{0}$ near the critical point. Parallel to Fig 8, Fig 11 shows the numerical dynamic factor as a function of the surfactant load in the asymmetric case. The amount of liquid-liquid interfaces is defined again by Eq. (55), but with $\hat{\phi}(\mathbf{r}, t):=2 \frac{\phi(\mathbf{r}, t)-\phi^{-}}{\phi^{+}-\phi^{-}}-1$, transforming the bulk values $\phi^{-}$and $\phi^{+}$to -1 and +1 , respectively $\left(\phi^{+}\right.$and $\phi^{-}$ were measured from the simulations here). According to Fig 11.b it is clear that the asymmetry has only marginal effect on the speed of phase separation compared to the symmetric case, which is in aggreement with Eq. (48), showing that $e$ is not in the leading order of $\kappa$. In addition, the marginal effect of the asymmetry applies on the entire range $\psi_{0} \in\left[0, \psi_{c}^{e=10}\right]$, where the critical point is only slightly shifted compared to the symmetric system, namely, $\psi_{c}^{e=10} \approx 0.0098$ has been found (we had equal dynamical factor $s=4 \times 10^{-4}$ for $\psi_{0}=0.00999$ and $\psi_{0}=0.0098$ in case of $e=0$ and $e=10$, respectively). Summarizing, it has been shown that asymmetry plays only a secondary role in the model, even near the critical point, which is only slighly shifted for moderate asym- 

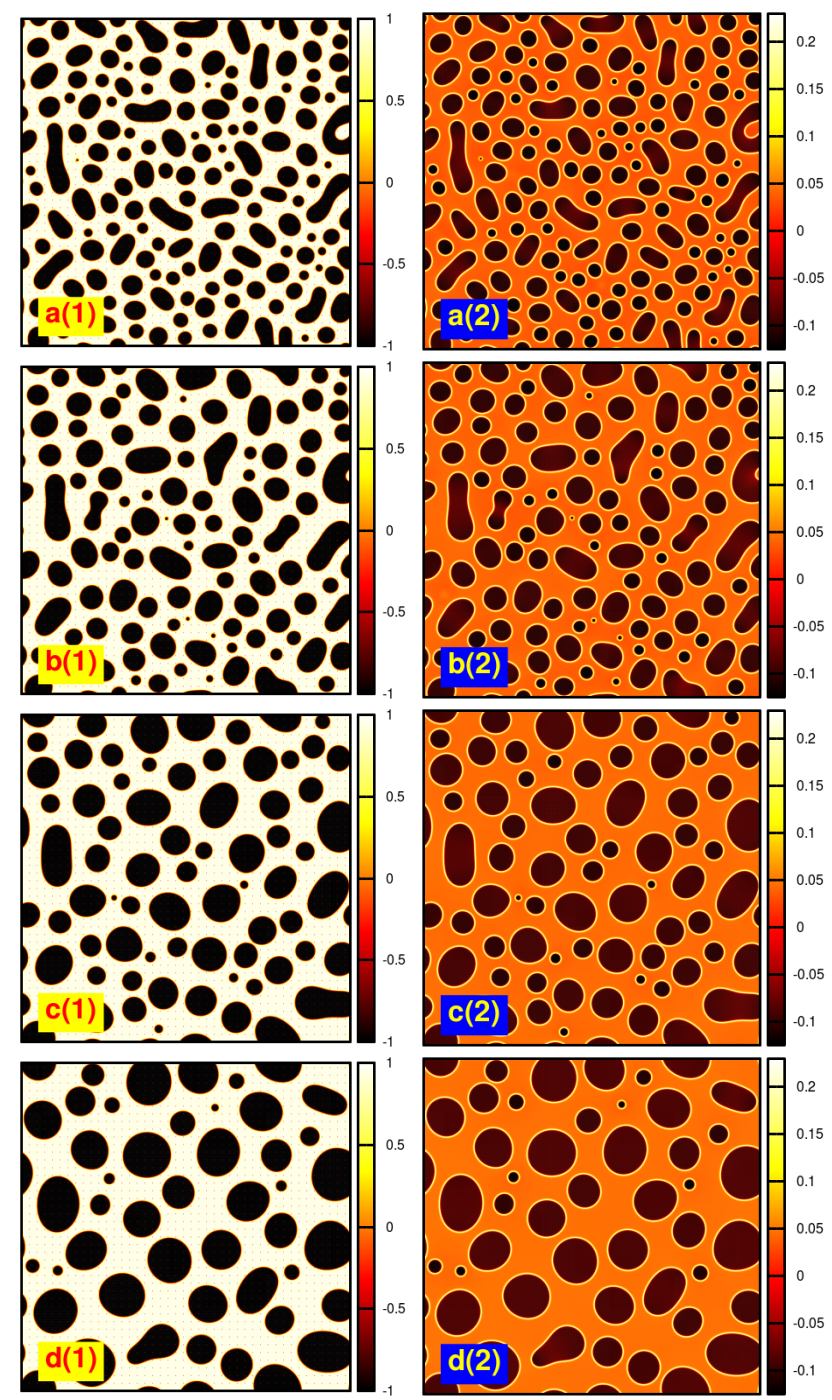

FIG. 9. Time evoltion of the asymmetric system for average surfactant load $\psi_{0}=0.005 . \phi(\mathbf{r}, t) / \phi_{0}$ and $\left[\psi(\mathbf{r}, t) / \psi_{0}\right]-1$ are shown on panels a(1)-d(1) and a(2)-d(2), respectively at $t=100,200,500$ and 1000 (time passes from top to bottom).

metry. Finally we mention that in case of assymetry the transient times have been found significantly smaller, i.e. pattern formation starts faster than in case of $e=0$. This is the reason why the time range $t=10 \ldots 100$ has been chosen for measuring the dynamic factor instead of $t=500 \ldots 1000$, as in case of $e=0$ (Practically we chose such ranges in which the $\log [Q(t)]$ vs $\log (t)$ lines were parallel to each other to make the measurement of $s$ possible). In this case the exponent $q=0.26$ emerges with $\Delta p=0.0,0.24288,0.45953,0.71155,1.06820,1.39520$ and 2.03370 , for the same average surfactant loads $\psi_{0} / \psi_{c}$ used in the symmetric case, respectively.
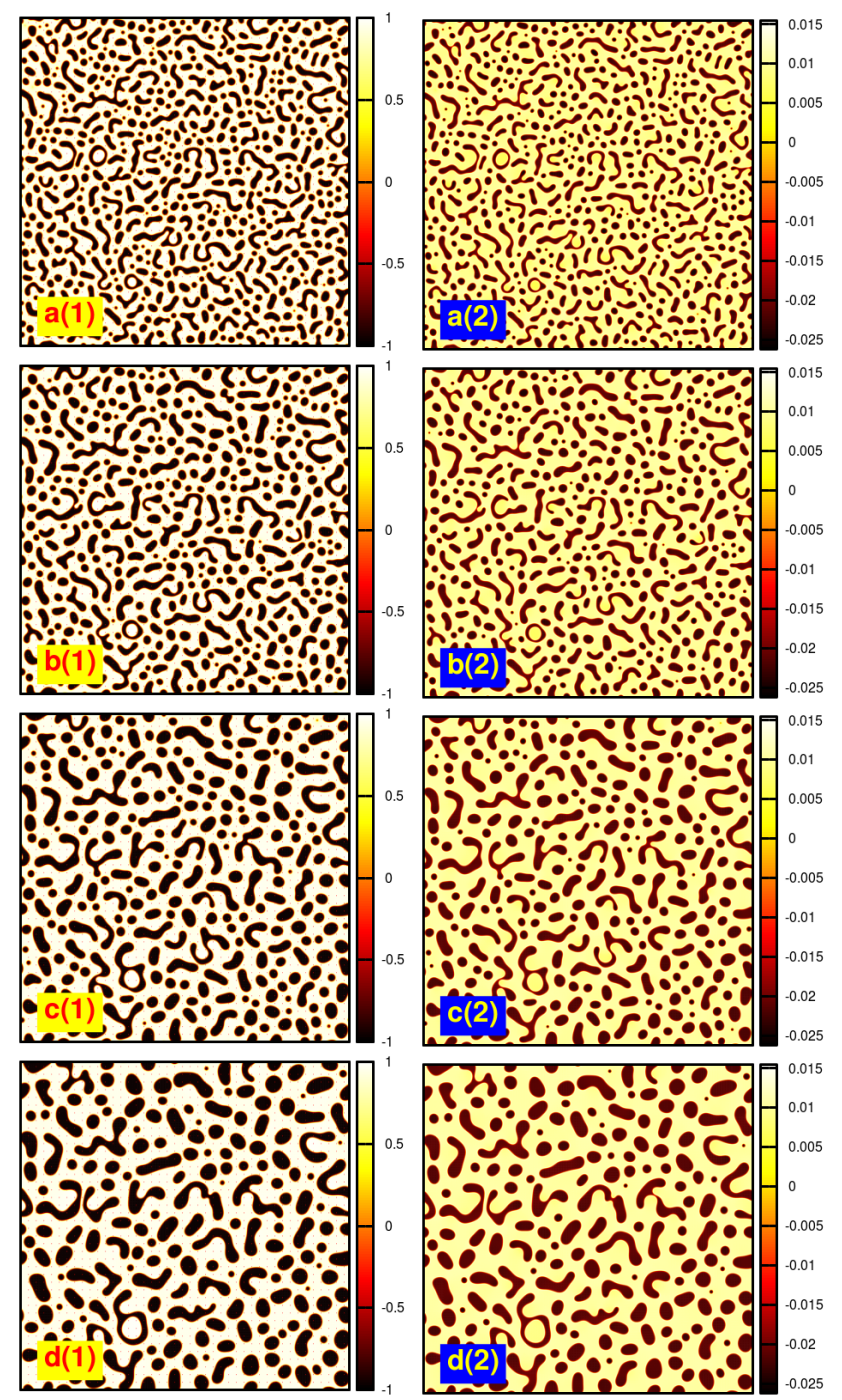

FIG. 10. Time evoltion of the asymmetric system for average surfactant load $\psi_{0}=0.0097 .2 \frac{\phi(\mathbf{r}, t)-\phi^{-}}{\phi^{+}-\phi^{-}}-1$ and $\left[\psi(\mathbf{r}, t) / \psi_{0}\right]-$ 1 are shown on panels a(1)-d(1) and a (2)-d(2), respectively at $t=100,200,500$ and 1000 (time passes from top to bottom).

\section{CONCLUSIONS}

In this work we've analyzed the Ginzburg-Landau free energy functional based generalized van der Sman / van der Graaf type diffuse interface model of surfactant assisted phase separation. We have shown that different regularization of the surface Dirac-delta function lead to different qualitative behavior in the speed of phase separation, equilibrium interfacial tension and interface width as a function of the surfactant load. The original, gradient-square regulariozation yields unphysical behavior of both the interface width and the speed of phase separation. In contrast, using the double-well function instead yields coherent physical picture, however, with divergent interface width at the critical point $\psi_{c}$ being 

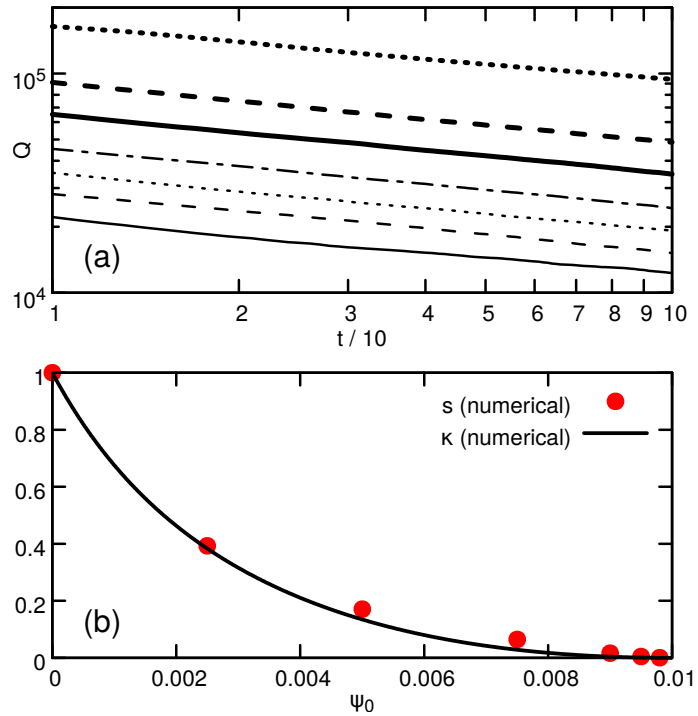

FIG. 11. Time evolution of the asymmetric system: (a) Amount of liquid-liquid interfaces as a function of time for different average surfactant loads. (b) Dynamic factor (or the relative speed of phase separation) defined by Eq. (57) (red dots) at surfactant loads corresponding to panel a, compared to the relative interfacial tension $\kappa$ obtained from the numerical solution of the Euler-Lagrange equations in case of $e=0$.

present in the general model (the citical point $\psi_{c}$ is a surfactant load where the interfacial tension vanishes). According to these, we proposed a hybrid regularization of the surface Dirac-delta function resulting in constant interface width but decreasing interfacial tension and phase separation speed as well, making the model numerically wieldy even near the critical point. Contrary to previous works, we analyzed the general model in the entire relevant surfactant load range, which reads as $\psi_{0} \in[0,1]$ for $\psi_{c}>1$ and $\psi_{0} \in\left[0, \psi_{c}\right]$ for $\psi_{c} \leq 1$, respectively. Fisrt we have shown that a realistic Langmuir/Frumkin isotherm emerges from the presence of the logarithmic therm, which is then must be used in physically consistent models. Second, since the absorbed amount of the surfactant at the interface (i.e. the interface load) is equal to the bulk value (far field load) at $\psi_{0}=\psi_{c}$, it has been proven that the approximations made in previous works for the Langmuir/Frumkin isotherms are valid only for $\psi_{c}>1$, however, the systems of interest may show even $\psi_{c} \ll 1$. In these cases, extended analysis is needed to investigate that qualitative behavior of the system when $\psi_{0} \lesssim \psi_{c}$. The analysis was based on a precise analytical derivation of the interfacial tension, which was then validated by numerical calculations in case of model parameters mimicking a real water/liquid $\mathrm{CO}_{2} /$ macromolecular surfactant system. The numerical results are in ecxellent agreement with the analytical calculations. Time dependent simulations have also been performed, showing that (i) the qualitative behavior of the system is not a function of the surfactant load, and (ii) the speed of the phase separation follows the reduction of the relative interfacial tension. It has also been shown that asymmetry (when bulk phases punish the presence of the surfactant field differently) enters the system only in the second order around $\psi_{0}=0$ and it has only minor effect either on the location of the critical point or on the speed of the phase separation as a function of the surfactant load. Finally we mention that the interfacial tension has been found to vanish as $\propto\left(\psi_{0}-\psi_{c}\right)^{2}$ near the critical point. In contrast, experiments clearly indicate $\propto \log \left(\psi_{0} / \psi_{c}\right)$ behavior in almost the entire range $\psi_{0} \in\left[0, \psi_{c}\right]$ [27], yielding linear relationship near $\psi_{c}$ instead of quadratic, suggesting that spontaneous emulsification is possible in these systems. Such a situation can be described either by changing the model parameters approriately to establish an emulsification point $\psi_{e}<\psi_{c}$, at which the interfacial tension vanishes, or applying nonlinear coupling of the surfactant field on the level of the free energy functional. Moreover, since maclomolecular surfactants result in the typical liquid-liquid interface width in the order of $0.1 \mu \mathrm{m}$, fluid flow may also play significant role in the time evolution of the system. One must not forget that the dynamical equations used in this study describe diffusioncontrolled processes, but avoid fast phase separation kinetics observed experimentally during liquid-liquid spinodal decomposition in binary systems. On other words, the liquid described by a pure diffusion type equation cannot flow, which must be then corrected appropriately. Nevertheless, there are different ways to introduce such correction. The phenomenological approach is to modify Fick's law to describe relaxing solute flux (finite speed of sound) instead of an instantenous one, thus introducing the second time derivative for the chemical concentration accounting for a wave mode [30, 31]. Another possibility is to simply apply the Navier-Stokes equation with an appropriate Korteweg pressure tensor, a problem which has been studied for binary $[32,33]$ and multicomponent $[34,35]$ systems, as well as in case of surfactant assisted liquid phase separation as mentioned in this paper before. Such a development, however, is the topic of a future study.

\section{ACKNOWLEDGEMENT}

This work has been supported by the VISTA basic research programme project No. 6359 "Surfactants for water $/ \mathrm{CO}_{2}$ /hydrocarbon emulsions for combined $\mathrm{CO}_{2}$ storage and utilization" of the Norwegian Academy of Science and Letters and the Statoil. We also thank professor S. Engblom (Uppsala University, Sweden) for the valuable discussions. 
[1] J. Sjoblom, Emulsions and Emulsion Stability: Surfactant Science Series/61, Surfactant Science (Taylor \& Francis, 1996).

[2] A. E. Hargreaves, Chemical Formulation, RSC Paperbacks (The Royal Society of Chemistry, 2003) pp. X001X004.

[3] D. Halpern, O. E. Jensen, and J. B. Grotberg, Journal of Applied Physiology 85, 333 (1998).

[4] B. A. Hills, Journal of Applied Physiology 87, 1567 (1999).

[5] D. Sarker, Pharmaceutical Emulsions: A Drug Developer's Toolbag (Wiley, 2013).

[6] D. Myers, Surfactant Science and Technology (Wiley, 2005).

[7] G. Ahearn, Journal of the American Oil Chemists Society 46, 540A (1969).

[8] S. Iglauer, Y. Wu, P. Shuler, Y. Tang, and W. A. G. III, Journal of Petroleum Science and Engineering 71, 23 (2010).

[9] S. Q. Tunio, A. H. Tunio, N. A. Ghirano, and Z. M. El Adawy, Intl. J. Appl. Sci. Tech. 1, 143 (2011).

[10] Z. Song, Z. Li, M. Wei, F. Lai, and B. Bai, Computers \& Fluids 99, 93 (2014).

[11] G. Gompper and S. Zschocke, Phys. Rev. A 46, 4836 (1992).

[12] O. Theissen and G. Gompper, The European Physical Journal B - Condensed Matter and Complex Systems 11, 91 (1999).

[13] T. Teramoto and F. Yonezawa, Journal of Colloid and Interface Science 235, 329 (2001).

[14] R. van der Sman and S. van der Graaf, Rheologica Acta 46, 3 (2006).

[15] H. Diamant and D. Andelman, Europhys. Lett. 34, 575 (1996).

[16] C.-H. Teng, I.-L. Chern, and M.-C. Lai, Discrete and Continuous Dynamical Systems B 17, 1289 (2012).
[17] H. Liu and Y. Zhang, Journal of Computational Physics 229, 9166 (2010).

[18] Li, Y. and Kim, J., Eur. Phys. J. B 85, 340 (2012).

[19] A. Yun, Y. Li, and J. Kim, Applied Mathematics and Computation 229, 422 (2014).

[20] S. Engblom, M. Do-Quang, G. Amberg, and A.-K. Tornberg, Commun. Comput. Phys. 14, 879 (2013).

[21] K. E. Teigen, P. Song, J. Lowengrub, and A. Voigt, Journal of Computational Physics 230, 375 (2011).

[22] W. H. Press, S. A. Teukolsky, W. T. Vetterling, and B. P. Flannery, Numerical recipes in $C$ (Cambridge University Press, 2002).

[23] G. Tegze, G. Bansel, G. I. Tóth, T. Pusztai, Z. Fan, and L. Gránásy, Journal of Computational Physics 228, 1612 (2009).

[24] L. C. Nielsen, I. C. Bourg, and G. Sposito, Geochimica et Cosmochimica Acta 81, 28 (2012).

[25] E. Torino, E. Reverchon, and K. P. Johnston, Journal of Colloid and Interface Science 348, 469 (2010).

[26] S. S. Adkins, X. Chen, I. Chan, E. Torino, Q. P. Nguyen, A. W. Sanders, and K. P. Johnston, Langmuir 26, 5335 (2010).

[27] M. Sagisaka, T. Fujii, Y. Ozaki, S. Yoda, Y. Takebayashi, Y. Kondo, N. Yoshino, H. Sakai, M. Abe, and K. Otake, Langmuir 20, 2560 (2004).

[28] W. Lu, H. Guo, I. Chou, R. Burruss, and L. Li, Geochimica et Cosmochimica Acta 115, 183 (2013).

[29] M. G. Davidson and W. M. Deen, Macromolecules 21, 3474 (1988).

[30] P. Galenko and D. Jou, Phys. Rev. E 71, 046125 (2005).

[31] P. Galenko and V. Lebedev, Physics Letters A 372, 985 (2008).

[32] G. Tegze, T. Pusztai, and L. Gránásy, Materials Science and Engineering: A 413414, 418 (2005), international Conference on Advances in Solidification Processes.

[33] G. Tegze and G. I. Tóth, Acta Materialia 60, 1689 (2012)

[34] J. Kim and J. Lowengrub, Int. Free Bound. 7, 435 (2005).

[35] J. Kim, Commun. Comput. Phys. 12, 613 (2012). 